\title{
Sex-specific distribution and classification of Wolbachia infections and mitochondrial DNA haplogroups in Aedes albopictus from the Indo-Pacific
}

Qiong Yang*1, Jessica Chung*1,2, Katie L. Robinson ${ }^{1}$, Thomas L. Schmidt ${ }^{1}$, Perran A. Ross ${ }^{1}$, Jiaxin Liang ${ }^{1}$ and Ary A. Hoffmann ${ }^{1}$

${ }^{1}$ Pest and Environmental Adaptation Research Group, Bio21 Institute and the School of BioSciences, The University of Melbourne, Parkville, VIC, Australia

${ }^{2}$ Melbourne Bioinformatics, The University of Melbourne, Parkville, VIC, Australia

* These authors contributed equally

Correspondence: ary@unimelb.edu.au (A.A.H.), qiongy@unimelb.edu.au (Q.Y.)

\section{Abstract}

The arbovirus vector Aedes albopictus (Asian tiger mosquito) is common throughout the Indo-Pacific region, where most global dengue transmission occurs. We analysed population genomic data and tested for cryptic species in 160 Ae. albopictus sampled from 16 locations across this region. We found no evidence of cryptic Ae. albopictus but found multiple intraspecific $\mathrm{COI}$ haplotypes partitioned into groups representing three Asian lineages: East Asia, Southeast Asia and Indonesia. Papua New Guinea (PNG), Vanuatu and Christmas Island shared recent coancestry, and Indonesia and Timor-Leste were likely invaded from East Asia. We used a machine learning trained on morphologically sexed samples to classify sexes using multiple genetic features and then characterized the $w A \mathrm{AlbA}$ and $w \mathrm{AlbB}$ Wolbachia infections in 664 other samples. The wAlbA and wAlbB infections as detected by qPCR showed markedly different patterns in the sexes. For females, most populations had a very high double infection incidence, with $67 \%$ being the lowest value (from Timor-Leste). For males, the incidence of double infections ranged from 100\% (PNG) to 0\% (Vanuatu). Only 6 females were infected solely by the wAlbA infection, while rare uninfected mosquitoes were found in both sexes. The wAlbA and wAlbB densities varied significantly among populations. For mosquitoes from Torres Strait and Vietnam, the wAlbB density was similar in single-infected and superinfected (wAlbA and wAlbB) mosquitoes. There was a positive association between wAlbA and wAlbB infection densities in superinfected $A e$. 
albopictus. Our findings provide no evidence of cryptic species of Ae. albopictus in the region and suggest site-specific factors influencing the incidence of Wolbachia infections and their densities. We also demonstrate the usefulness of SNPs as sex-specific mosquito markers. The results provide baseline data for the exploitation of Wolbachia-induced cytoplasmic incompatibility $(\mathrm{Cl})$ in dengue control.

\section{Keywords}

Aedes albopictus, Dengue, mosquito sexing, Wolbachia, superinfection, cryptic species

\section{Introduction}

The mosquito Aedes albopictus is an important disease vector, capable of transmitting dengue, chikingunya and other arboviruses. As such, it has been targeted for control in many countries around the world, including areas where it has invaded relatively recently from its origin in east Asia such as North America and Europe [1]. Aedes albopictus is common throughout the Indo-Pacific region, where $70 \%$ of global dengue transmission occurs [2]. Control is challenging due to a variety of factors including the evolution of pesticide resistance $[3,4]$ and the wide range of hosts and habitats used by Ae. albopictus [5], which has led to renewed interest in developing alternative methods of control and disease suppression. These include using the endosymbiotic bacterium Wolbachia, where males carrying a novel Wolbachia infection are released to sterilise females [6-8], or to spread through populations to reduce arboviral transmission by the mosquitoes $[9,10]$. When applying Wolbachia technology, it is important to understand both the taxonomic identity of the mosquito target as well as the status of natural Wolbachia infections in the mosquito target. Both factors are important in the case of Ae. albopictus, as a cryptic Ae. albopictus subspecies has been described from China [11] and Vietnam [12] and because Ae. albopictus in the field naturally harbor the Wolbachia strains wAlbA or wAlbB (and are often superinfected with both strains) [13,14]. Patterns of population genetic structure can indicate areas where mosquito genetic backgrounds are likely to be similar or different to 
those of target populations [15] - an important consideration for widespread species such as Ae. albopictus that can be locally adapted to different conditions [16].

Both suppression and invasion of populations by novel Wolbachia depend on interactions between the novel strain and extant Wolbachia strains as well as their impacts on host fitness and transmission efficiency [17]. In Ae. albopictus, wAlbA and wAlbB have been reported as increasing host fecundity [18]. Both wAlbA and wAlbB infections also induce cytoplasmic incompatibility $(\mathrm{Cl})$, the phenomenon where males carrying a particular Wolbachia strain are incompatible with females lacking that strain, resulting in the production of unviable embryos, which is a common phenotype in insects [19]. Females infected with only wAlbA or wAlbB strain show $\mathrm{Cl}$ when crossed with superinfected males resulting in unidirectional $\mathrm{Cl}$ [13]. However, this $\mathrm{Cl}$ phenotype may be rarely expressed in nature if there is a high frequency of individuals carrying both wAlbA and wAlbB and a low level of polymorphism within these infections [20]. Other factors influencing the incidence of these infections in natural populations include maternal transmission efficiency which can be imperfect for Wolbachia [19] and may be somewhat lower for the wAlbA strain than for wAlbB (estimates of 97.5 versus $99.6 \%$, respectively for one region) [14]. Both fitness effects and transmission of Wolbachia may relate partly to Wolbachia density; high densities of endosymbionts are more likely to decrease host fitness but maintain a high level of maternal transmission and $\mathrm{Cl}$ [17]. Moreover, variability in density is important because crosses between individuals with the same strain but different densities can produce $\mathrm{Cl}$ [21].

In this paper, we build on existing work to provide an overview of wAlbA and wAlbB infections among Ae. albopictus in the Indo-Pacific region. We aim to characterise the incidence of these infections from field collections across the region. We also test for the cryptic species status of the collections given that Wolbachia may be absent from one of the cryptic subspecies [12]. We focus on any sex-related differences in infection frequencies given that the incidence of WAlbA in particular may differ between males and females [22, 23]. To test associations between Wolbachia and sex in samples that were only available to us without sexing (e.g. larval samples, DNA samples), we develop a method based on previously collected SNP data to sex the mosquitoes given that the sexes of Aedes mosquitoes can be differentiated through SNPs at multiple pseudosex regions [24]. Finally, we consider variation in the field density of the Wolbachia infections to test whether there 
are possible interactions between the infections given that the presence of one endosymbiont in an invertebrate host can influence the density of another endosymbiont [25]. We discuss our results with respect to future Wolbachia-based strategies against Ae. albopictus.

\section{Material and Methods}

\section{Sample collection}

Aedes albopictus were sampled from 17 locations from Mauritius to Fiji to Japan (Fig. 1, Table 1). We considered mosquitoes collected within the same country to be from the same population. Genome sequencing data obtained from a total of 664 Ae. albopictus individuals among these populations have been used for population genetic analysis in our previous study [15]. Here, we used SNPs to predict sex and classify Wolbachia infection status by sex (see below).

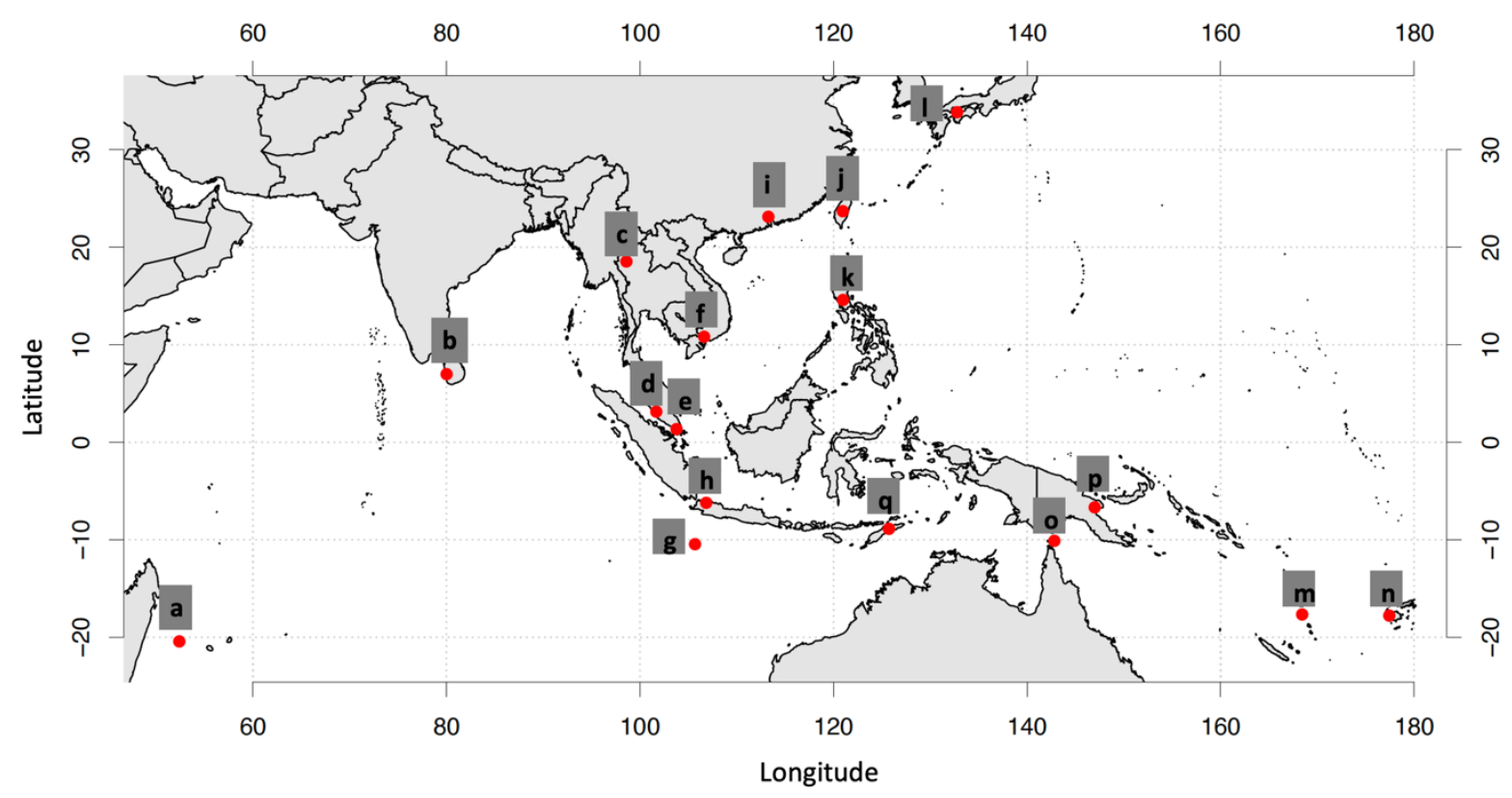

Figure 1. Approximate locations of the 17 Aedes albopictus populations. Each letter corresponds to a collection from a different country. Note that in some cases multiple samples from nearby locations were combined (Table 1).

Table 1 Details of Aedes albopictus sampled from 17 populations. See Figure 1 for map ID locations. 


\begin{tabular}{|c|c|c|c|c|}
\hline Map ID & Country & $\begin{array}{c}\text { Year(s) } \\
\text { collected }\end{array}$ & Locations combined & Life stage \\
\hline a & Mauritius & 2017 & & adult \\
\hline$b$ & Sri Lanka & 2017 & & larva \\
\hline C & Thailand & 2016 & & adult \\
\hline$d$ & Malaysia & $2015 / 2017$ & $\begin{array}{c}\text { Pahang, Johor, } \\
\text { Selangor and Perlis }\end{array}$ & adult \\
\hline e & Singapore & 2015 & & adult \\
\hline$f$ & Vietnam & 2014 & & adult \\
\hline g & Christmas Island & 2018 & & larva \\
\hline $\mathrm{h}$ & Indonesia & $2016 / 2017$ & $\begin{array}{c}\text { Jakarta, Bandung } \\
\text { and Bali }\end{array}$ & $\begin{array}{l}\text { Jakarta and Bandung: } \\
\text { adult, Bali: larva }\end{array}$ \\
\hline $\mathrm{i}$ & China & 2017 & & adult \\
\hline j & Taiwan & 2016 & & most likely adults \\
\hline k & Philippines & 2016 & & most likely adult \\
\hline I & Japan & 2015 & & adult \\
\hline $\mathrm{m}$ & Vanuatu & 2018 & & most likely adults \\
\hline $\mathrm{n}$ & Fiji & 2018 & & most likely adult \\
\hline 0 & Torres Strait & 2018 & multiple islands & adult \\
\hline $\mathrm{p}$ & $\begin{array}{c}\text { Papua New Guinea } \\
\text { (PNG) }\end{array}$ & 2019 & $\begin{array}{l}\text { Port Moresby and } \\
\text { Madang }\end{array}$ & adult \\
\hline$q$ & Timor-Leste & 2019 & & adult \\
\hline
\end{tabular}

\section{Mitochondrial COI as a DNA barcode}

The universal barcode region of the $\mathrm{CO} 1$ gene ( $658 \mathrm{bp}$ ) of individual Ae. albopictus was amplified using the common primers (LCO1490 5' GGTCAACAAATCATAAAGATATTGG

\section{3' and HCO2198 5' TAAACTTCAGGGTGACCAAAAAATCA 3') [26]. Amplifications were} performed in a Thermal Cycler (Eppendorf, Germany) with an adjusted annealing temperature of $55^{\circ} \mathrm{C}$. PCR amplicons from individuals were sequenced in both forward and reverse directions using Sanger Sequencing (Macrogen, Inc., Geumcheongu, Seoul, South Korea). The trimmed 623 bp sequence was analysed with Geneious 9.18 software (Kearse et al., 2012) to investigate SNP variation among samples.

The CO1 gene sequencing data obtained from 160 mosquitoes were analysed with the Molecular Evolutionary Genetics Analysis (MEGA) program version 7.0 (Tamura et. al., 2013). A phylogenetic tree was constructed with a neighbour-joining model applied to a 
genetic distance matrix with the Kimura-2 parameter model implemented with 1000 bootstrap replications in MEGA.

\section{ML sex classification with ddRAD-seq}

To build a machine learning $(\mathrm{ML})$ classifier, we used a semi-supervised learning approach with 91 individuals from Torres Strait that had both sexing based on morphology and ddRAD sequencing data, and 134 unsexed samples with ddRAD sequencing data only. For each sample BWA-MEM v0.7.17 [27] was used to map sequencing reads to the Ae. albopictus reference genome (GenBank accession no. GCA_006496715.1) and determined the relative sequencing depths of $26,782100 \mathrm{kbp}$ sized regions. For each of these $100 \mathrm{kbp}$ regions, a linear model was fit to identify if sex had a significant effect on depth, using sex and sequencing batch as predictor variables. We identified 85 regions with significant sex effects after multiple hypothesis testing correction. A PCA plot using the sequencing depths of significant regions showed two distinct clusters (Fig. S1a), however, we observed 15 individuals not clustering with their expected group suggesting some samples may have been assigned the incorrect sex. These samples were removed from the training set. Using the e1071 R library [28], a preliminary SVM model was trained using the significant regions as features and the 135 unsexed samples were classified using this model (Fig. S1b).

For the purposes of creating a stand-alone program for classifying new ddRAD samples, we used samples with classification probability $>70 \%$ from the preliminary model and extracted candidate ddRAD tags within the identified $100 \mathrm{kbp}$ regions as candidate features. From these, we used linear models to identify 42 ddRAD tag features that had their sequencing depths associated with sex. In addition, 109 negative control ddRAD tags not associated with sex were included for sequence depth normalisation purposes. A SVM model was trained using the e1071 library and the classifier has been made available on GitHub (https://github.com/pearg/albo_spm).

To validate the model, we obtained an additional 127 sexed individuals from different populations (Indonesia, Japan, Vietnam, and Torres Strait). 124 of these had sufficient sequencing depth to predict sex, and we compared the sex predicted from our model to the sex obtained from morphology. A confusion matrix (predicted versus actual cases) was used 
to test the quality of the classification model with a Matthews correlation coefficient computed to quantify the agreement.

\section{Wolbachia infection classification with ddRAD-seq}

Due to insufficient observations of uninfected samples and samples infected with wAlbA only, we limited our classifier to single wAlbB infected samples and superinfected (wAlbA and wAlbB) samples. To build the Wolbachia strain classifier, we first obtained SNP sites between the wAlbA strain and the wAlbB strain by comparing the Wolbachia wAlbA FL2016 strain contig-level assembly (GenBank accession no. GCA_002379155.2) to the wAlbB complete assembly (GenBank accession no. GCA_004171285.1) by simulating 100bp reads from the $w A \mathrm{AlbA}$ reference and mapping them to the $w \mathrm{AlbB}$ reference. Using only the uniquely mapping reads, we used samtools mpileup [29] to obtain sites with single nucleotide differences between the two references, resulting in 9,697 sites.

Wolbachia-infected Ae. albopictus ddRAD-seq samples were aligned using Bowtie2 v2.3.4.3 [30] to the $w \mathrm{AlbB}$ reference assembly and variant calling was performed with FreeBayes v1.3.5 [31]. We then selected SNPs that were previously identified in the wAlbA/wAlbB reference assembly comparison, resulting in 649 SNP sites. Samples with fewer than 10 allelic observations were removed, leaving 478 wAlbB infected $(n=63)$ or superinfected $(n=$ 415 ) samples. The data was split into a training and test set of $80 \%$ and $20 \%$ respectively.

For each sample, a score was created using the number of observed wAlbA alleles divided by the total number of alleles observed, then Laplace smoothed with a pseudocount of 1 . A univariate logistic regression model was built using the natural log of the score. The test dataset was used to assess the performance of the model. As with the sexing prediction, a confusion matrix (predicted versus actual cases) was used to test the performance of the classification model.

\section{Wolbachia detection via qPCR assay}

For real-time PCR detection, we used a LightCycler ${ }^{\circledR} 480$ High Resolution Melting Master (HRMM) kit (Roche; Cat. No. 04909631001, Roche Diagnostics Australia Pty. Ltd., Castle Hill New South Wales, Australia) and IMMOLASETM DNA polymerase (5 U/ $\mu$ ) (Bioline; Cat. No. BIO-21047) as described by Lee et al. (2012) [32]. The PCR conditions for DNA amplification 
began with a 10 -minute pre-incubation at $95^{\circ} \mathrm{C}$ (Ramp Rate $\left.=4.8^{\circ} \mathrm{C} / \mathrm{s}\right)$, followed by 40 cycles of $95^{\circ} \mathrm{C}$ for 5 seconds (Ramp Rate $=4.8^{\circ} \mathrm{C} / \mathrm{s}$ ), $53^{\circ} \mathrm{C}$ for 15 seconds (Ramp Rate $=2.5^{\circ} \mathrm{C} / \mathrm{s}$ ), and $72^{\circ} \mathrm{C}$ for 30 seconds (Ramp Rate $=4.8^{\circ} \mathrm{C} / \mathrm{s}$ ).

One set of Ae. albopictus-specific primers was used to confirm that individual mosquitoes were correctly identified as Ae. albopictus and as a qPCR positive control. Primer sets specific to either wAlbA or WAlbB were used to infer the presence or absence of wAlbA and wAlbB infection (wAlbB1 CCTTACCTCCTGCACAACAA, GGATTGTCCAGTGGCCTTA wAlbA-F GTAGTATTTACCCCAGCAG QArev2 CACCAGCTTTTAACTTGACC) [33]. Crossing point (Cp) values of three consistent replicate runs were averaged to produce the results. Differences in $\mathrm{Cp}$ values between the Ae. albopictus marker and the wAlbA and wAlbB markers were transformed by $2^{\mathrm{n}}$ to produce relative Wolbachia density measures.

\section{Statistical analyses}

We compared the proportion of superinfected individuals among the sexes and populations using a generalized linear model with a binomial distribution. We also directly compared the distribution of superinfected and wAlbB singly infected individuals across sexes in some populations with relatively larger sample sizes, treating these as contingency tables. All these analyses were run in IBM Statistics SPSS version 26.

For the Wolbachia density data, we examined variation in density across populations and sexes following logarithmic transformation of the data for normality, focussing on those individuals carrying both infections. We also examined associations between wAlbA and wAlbB infection density at the individual level within each population by computing Pearson's correlations on logarithmically transformed densities and treating the sexes separately given the density differences observed between the sexes (see below). Linear regressions were also computed to see if the density of wAlbA could predict that of wAlbB. We only considered correlations and regressions for populations where data from at least 10 individuals were available. Finally, we tested if there was a difference between wAlbB densities in singly and superinfected females and males; we focussed on the Torres Strait and Vietnam populations, where samples falling into both categories were available, and included sex and infection type as factors for Torres Strait and only infection type in Vietnam since only females had sufficient numbers of each infection type. 


\section{Results}

\section{Variation in Ae. albopictus}

PCR amplification and sequencing of the mtDNA CO1 gene resulted in a $623 \mathrm{bp}$ fragment for each individual Ae. albopictus with no insertions or deletions. A total of 23 haplotypes were identified from 160 individuals collected in the Indo-Pacific region. Haplotype diversity varied for each region with Indonesia (31 individuals, 8 haplotypes), Vietnam (16 individuals, 6 haplotypes) and Thailand ( 8 individuals, 5 haplotypes) having substantially higher haplotype diversities than Taiwan (14 individuals, 3 haplotypes), Sri Lanka (14 individuals, 2 haplotypes), Malaysia (12 individuals, 2 haplotypes), PNG (11 individuals, 2 haplotypes), Singapore ( 8 individuals, 2 haplotypes) and Vanuatu (19 individuals, 1 haplotype).

Three predominant haplotypes were identified in Ae. albopictus populations: H1 (17.5\%) detected in Vanuatu, PNG and Christmas Island, suggesting recent co-ancestry; H2 (15.0\%) detected in Indonesia, Torres Strait, Timor-Leste and Philippines; and H11 (18.8\%) detected in a wide range of populations, including Malaysia, Singapore, Thailand, Indonesia, Fiji, Philippines, Vietnam and PNG. Other haplotypes were either unique to a specific population or had a limited geographical coverage.

To determine the relationships among samples, we constructed a median-joining network using haplotypes based on sequence variation. Haplotypes were connected when the probability of parsimony was at least 95\%. The COI haplotype network (Fig. 2) suggested some mitochondrial genetic structure between regions. The spatial distribution of ancestral lineages among Ae. albopictus can be interpreted with reference to the native and invasive ranges of this species. A network analysis of Ae. albopictus partitioned haplotypes into three haplogroups representing three native range lineages. The northernmost of these (red dotted circle) showed a common heritage among East Asian populations. This lineage was also dominant in Mauritius. The second native range lineage (blue dotted circle) had a common heritage among Southeast Asian populations (excluding Indonesia), and was also found in Christmas Island, Fiji, PNG and Vanuatu. Differentiation was low between these lineages. The Indonesian Ae. albopictus lineage (green dotted circle) was distinct from the East and Southeast Asian native range lineages and was also found in the Torres Strait 
Islands and Timor-Leste. The Philippine haplotypes were split into two groups, Southeast Asia and Indonesia. This likely reflects the Philippine population as having ancestry from both groups. A further split was observed in the phylogenetic tree based on $\mathrm{CO} 1$ sequence variation from the 23 haplotypes in this study, the 2 Ae. albopictus cryptic haplotypes (KY765450.1 and KY765459) [11], as well as the outgroup Ae. scutellaris (KP843372, Fig. 3). The maximum divergence observed in the 23 haplotypes was 1.6\% (Table S1), significantly lower than interspecific divergence (12.8\%), indicating that cryptic Ae. albopictus are not present across the samples of the Indo-Pacific region tested here.
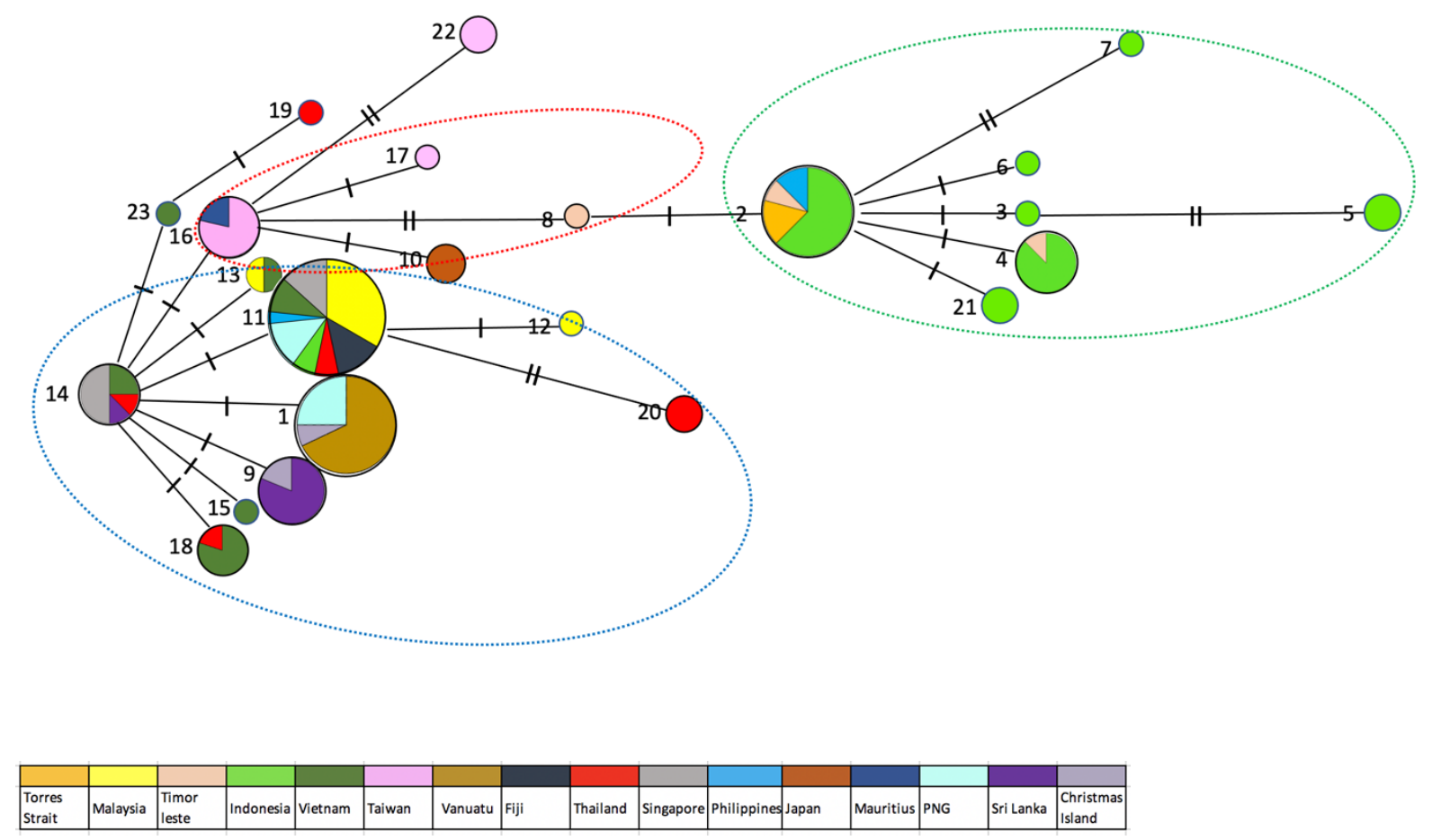

Figure 2. Haplotype network for COI. Each coloured node represents an observed haplotype with circle size indicating the number of individuals with each numbered haplotype and the slash along the connecting lines indicating the number of nucleotide differences. 


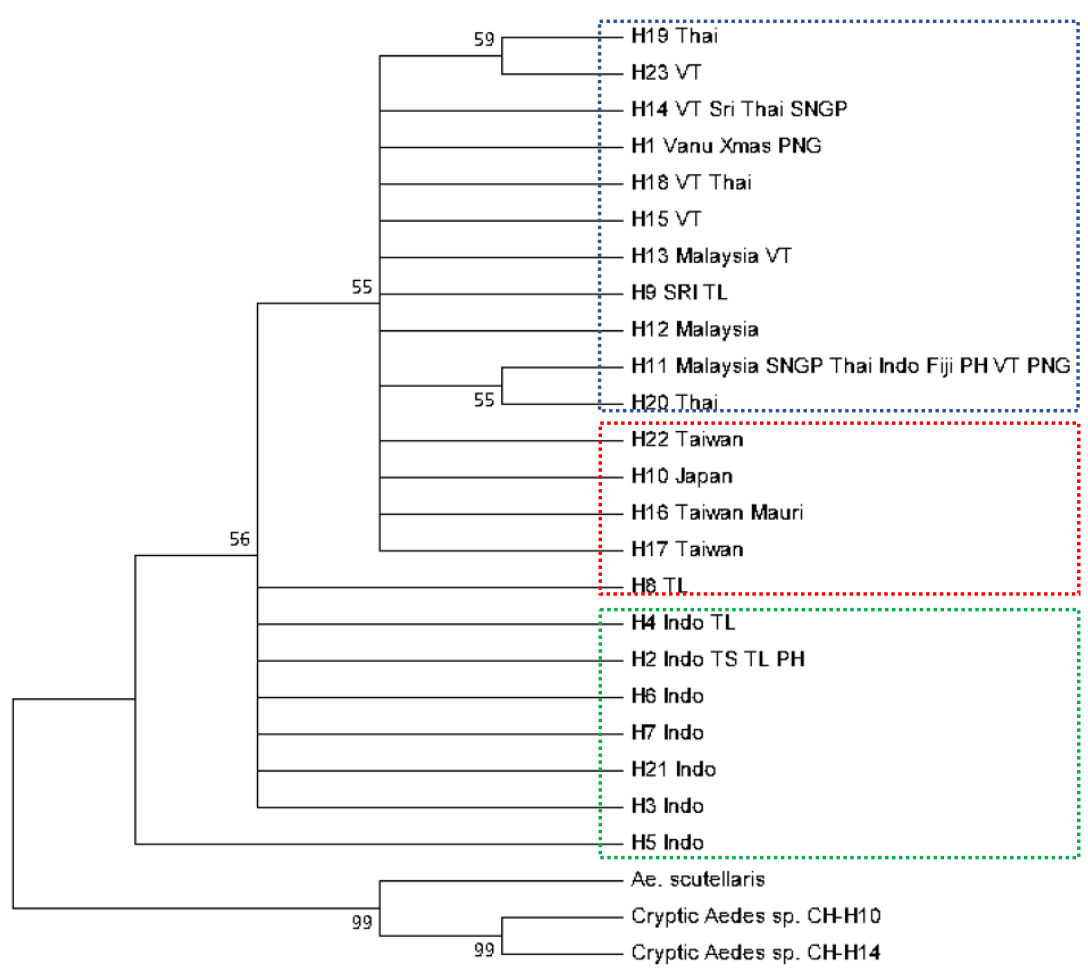

Figure 3. Phylogenetic analysis based on $\mathrm{CO} 1$ haplotype variation. Neighbor-joining trees constructed via Kimura-2 parameter model using MEGA. Numbers at branches represent bootstrap values of 1000 replicates (values $>50$ are shown). Sequences from different outgroup species of the genus Aedes were selected from GenBank (Ae. albopictus cryptic haplotypes KY765450.1 and KY765459 and Ae. scutellaris KP843372). Abbreviations: Thai, Thailand; VT, Vietnam; Sri, Sri Lanka; SNGP, Singapore; Vanu, Vanuatu; Xmas, Christmas Island; PNG, Papua New Guinea; TL, Timor-Leste; TS, Torres Strait; Indo, Indonesia; PH, Philippines.

\section{Sex Determination}

As shown in Table 2, the classification performance on the 124 samples in the test set yielded a model accuracy of $97.6 \%$ with three incorrect classifications. The model had a Matthews correlation coefficient (MCC) of 0.940 .

Table 2. Test set confusion matrix for sex prediction of mosquitoes based on SNP markers identified from a Torres Strait dataset but tested against sexed samples from other populations. 
Predicted sex

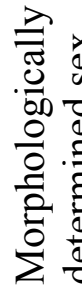

Female Male

Female

Male

\begin{tabular}{|c|c|}
\hline 89 & 3 \\
\hline 0 & 32 \\
\hline
\end{tabular}

We then used our model to classify a further 510 samples without morphological sexing (Table 4, excluding Torres Strait samples).

\section{Wolbachia infection classification}

As shown in Table 3, the classification performance of the 95 samples in the test set yielded a model accuracy of $87.3 \%$. However, the dataset was imbalanced with most samples being superinfected, thus only yielding an MCC of 0.634 . The majority of the discordant samples were predicted to be superinfected but were only scored as singly infected with wAlbB in the qPCR assay, producing the low MCC value. We suspect that this relates to the limit of detection of wAlbA in the qPCR assay rather than inaccurate classification of the samples (see discussion).

Table 3. Test set confusion matrix of Wolbachia infection classification for wAlbB and superinfected (wAlbA and wAlbB) individuals from different populations, with values scored from the qPCR assay being predicted by the ML SNP-based method.

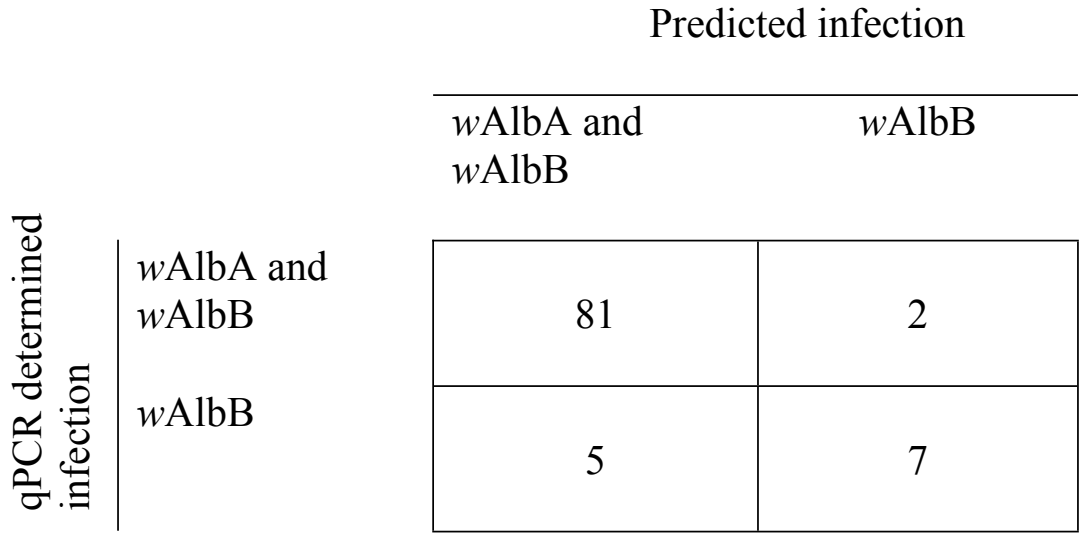


A total of 664 Ae. albopictus individuals among 15 populations were processed and screened for the presence of Wolbachia using qPCR. Overall, wAlbA and wAlbB infections showed markedly different patterns with regard to sex (Table 4). The incidence of superinfected individuals was almost always higher in females than in males. For females, most of the populations had a very high superinfection incidence, with 6 populations showing a superinfection incidence of $100 \%$ in females: Taiwan, Thailand, Singapore, Philippines, Japan and Mauritius. The lowest incidence of superinfection (67\%) was in females from Timor-Leste. There was substantial variation in the incidence of superinfections in males detected by qPCR. Excluding populations which had sample sizes less than 3, the PNG population had the highest superinfection incidence in males (100\%) while Vanuatu had the lowest incidence (0\%). When we analysed the populations with the largest sample sizes for each sex and considered only the wAlbB and superinfected individuals ( $\mathrm{N}>5$ per sex), a generalized linear model indicated a significant interaction among sex and population $\left.X^{2}=1493.2, d f=4, P<0.001\right)$. In each population, the frequency of individuals infected with only wAlbB was lower in females than in males and sex differences were significant $(P<0.05)$ in four of the five populations by contingency tests. We also found 6 females that were singly infected with wAlbA, while rare uninfected $(0 \%$ 6.7\%) mosquitoes were equally likely to occur in either sex.

Table 4. Wolbachia infection status in Ae. albopictus females and males as assessed by qPCR screening. Sexes were identified morphologically or through the ML sequencing-based approach as indicated.

\begin{tabular}{|c|c|c|c|c|c|c|}
\hline \multirow{2}{*}{ Population } & \multirow{2}{*}{ Sex } & \multicolumn{4}{|c|}{ Infection status (\%) } & \multirow{2}{*}{$\begin{array}{l}\text { Number of } \\
\text { individuals }\end{array}$} \\
\hline & & $w A l b A$ & wAlbB & $\begin{array}{c}\text { wAlbA and } \\
\text { wAlbB }\end{array}$ & Uninfected & \\
\hline \multirow{2}{*}{$\begin{array}{c}\text { Torres Strait } \\
\text { (multiple islands) }\end{array}$} & Male (M) & 0 & 52.4 & 47.6 & 0 & 63 \\
\hline & Female (M) & 0 & 12.2 & 87.7 & 1.1 & 90 \\
\hline \multirow{2}{*}{$\begin{array}{l}\text { Malaysia (Pahang, } \\
\text { Johor, } \\
\text { Selangor and Perlis) }\end{array}$} & Male (P) & 0 & 7.4 & 88.9 & 3.7 & 27 \\
\hline & Female $(\mathrm{P})$ & 0 & 1.1 & 97.8 & 1.1 & 89 \\
\hline \multirow{2}{*}{ Timor-Leste } & Male (M) & 0 & 28.6 & 71.4 & 0 & 14 \\
\hline & Female (M) & 11.1 & 15.5 & 66.7 & 6.7 & 45 \\
\hline Indonesia (Jakarta, & Male (P) & 0 & 50 & 50 & 0 & 6 \\
\hline
\end{tabular}




\begin{tabular}{|c|c|c|c|c|c|c|}
\hline $\begin{array}{l}\text { Bandung } \\
\text { and Bali) }\end{array}$ & Female $(\mathrm{P})$ & 0 & 0 & 100 & 0 & 36 \\
\hline \multirow{2}{*}{$\begin{array}{l}\text { Vietnam (Ho Chi } \\
\text { Minh City) }\end{array}$} & Male (M) & 0 & 50 & 50 & 0 & 2 \\
\hline & Female (M) & 0 & 22.2 & 72.2 & 5.6 & 18 \\
\hline \multirow{2}{*}{ Taiwan } & Male (P) & 0 & 62.5 & 37.5 & 0 & 8 \\
\hline & Female $(\mathrm{P})$ & 0 & 0 & 100 & 0 & 10 \\
\hline \multirow{2}{*}{ Vanuatu } & Male (P) & 0 & 100 & 0 & 0 & 9 \\
\hline & Female (P) & 0 & 14.3 & 85.7 & 0 & 7 \\
\hline Fiji (Nadi) & Female $(\mathrm{P})$ & 0 & 12.5 & 87.5 & 0 & 16 \\
\hline \multirow{2}{*}{$\begin{array}{l}\text { Thailand (Chiang Mai, } \\
\text { BKK) }\end{array}$} & Male (P) & 0 & 0 & 0 & 100 & 2 \\
\hline & Female $(\mathrm{P})$ & 0 & 0 & 100 & 0 & 7 \\
\hline Singapore & Female $(\mathrm{P})$ & 0 & 0 & 100 & 0 & 8 \\
\hline \multirow{2}{*}{ Philippines (Manlia) } & Male (P) & 0 & 0 & 100 & 0 & 1 \\
\hline & Female $(\mathrm{P})$ & 0 & 0 & 100 & 0 & 6 \\
\hline \multirow{2}{*}{ Japan (Matsuyama) } & Male (P) & 0 & 33.3 & 66.7 & 0 & 3 \\
\hline & Female $(\mathrm{P})$ & 0 & 0 & 100 & 0 & 3 \\
\hline \multirow{2}{*}{ Mauritius } & Male (P) & 0 & 50 & 50 & 0 & 2 \\
\hline & Female $(\mathrm{P})$ & 0 & 0 & 100 & 0 & 4 \\
\hline China (Guangzhou) & Female $(\mathrm{P})$ & 0.7 & 1.3 & 98 & 0 & 151 \\
\hline \multirow{2}{*}{$\begin{array}{l}\text { PNG (Port Moresby } \\
\text { and Madang) }\end{array}$} & Male $(P)$ & 0 & 0 & 100 & 0 & 3 \\
\hline & Female (P) & 0 & 0 & 97 & 3.0 & 33 \\
\hline
\end{tabular}

Abbreviation: $M$, sexed based on morphology; $P$, sexed based on prediction using the $M L$ model.

Similar patterns to those presented in Table 4 were detected when the wAlbB and superinfection was identified through SNPs (Table 5). Although numbers are lower, these results also highlight the higher incidence of the superinfection in females compared to males in several populations.

Table 5. Sex and strain distribution of Wolbachia infection status in Ae. albopictus using classification results. Only samples with $>60 \%$ classification probability in both sex and Wolbachia strain prediction are included.

\begin{tabular}{|c|c|c|c|c|}
\hline \multirow[b]{2}{*}{ Population } & \multirow[b]{2}{*}{$\begin{array}{l}\text { Predicted } \\
\text { Sex }\end{array}$} & \multicolumn{3}{|c|}{ Number of individuals } \\
\hline & & $\begin{array}{l}\text { Predicted } \\
\text { wAlbB }\end{array}$ & $\begin{array}{c}\text { Predicted } \\
\text { wAlbA and } \\
w A l b B\end{array}$ & Total \\
\hline \multirow{2}{*}{ China (Guangzhou) } & Female & 0 & 151 & 151 \\
\hline & Male & 0 & 1 & 1 \\
\hline \multirow{2}{*}{ Indonesia (Bandung) } & Female & 0 & 12 & 12 \\
\hline & Male & 0 & 2 & 2 \\
\hline Indonesia (Bali) & Female & 0 & 8 & 8 \\
\hline
\end{tabular}




\begin{tabular}{|c|c|c|c|c|}
\hline & Male & 0 & 2 & 2 \\
\hline \multirow{2}{*}{ Indonesia (Jakarta) } & Female & 0 & 17 & 17 \\
\hline & Male & 0 & 0 & 0 \\
\hline \multirow{2}{*}{ Japan (Matsuyama } & Female & 0 & 5 & 5 \\
\hline & Male & 0 & 1 & 1 \\
\hline \multirow{2}{*}{$\begin{array}{l}\text { Malaysia (Pahang, } \\
\text { Johor, } \\
\text { Selangor and Perlis) }\end{array}$} & Female & 0 & 61 & 61 \\
\hline & Male & 0 & 15 & 15 \\
\hline \multirow{2}{*}{ Mauritius } & Female & 0 & 3 & 3 \\
\hline & Male & 2 & 0 & 2 \\
\hline \multirow{2}{*}{ Fiji (Nadi) } & Female & 0 & 16 & 16 \\
\hline & Male & 0 & 0 & 0 \\
\hline \multirow{2}{*}{ Philippines } & Female & 0 & 6 & 6 \\
\hline & Male & 0 & 1 & 1 \\
\hline \multirow{2}{*}{ Singapore } & Female & 0 & 8 & 8 \\
\hline & Male & 0 & 0 & 0 \\
\hline \multirow{2}{*}{ Sri Lanka } & Female & 0 & 0 & 0 \\
\hline & Male & 0 & 1 & 1 \\
\hline \multirow{2}{*}{ Taiwan } & Female & 1 & 8 & 9 \\
\hline & Male & 4 & 1 & 5 \\
\hline \multirow{2}{*}{ Torres Strait } & Female & 0 & 123 & 123 \\
\hline & Male & 38 & 25 & 63 \\
\hline \multirow{2}{*}{ Vanuatu } & Female & 0 & 7 & 7 \\
\hline & Male & 4 & 3 & 7 \\
\hline \multirow{2}{*}{ Vietnam } & Female & 0 & 6 & 6 \\
\hline & Male & 1 & 0 & 1 \\
\hline
\end{tabular}

\section{Wolbachia density comparisons}

Wolbachia density was influenced by sex and the location where samples were collected

(Fig 4). For wAlbA density, there was a significant effect of $\operatorname{sex}\left(F_{(1,345)}=38.800, P=0.001\right)$ and population $\left(\mathrm{F}_{(12,345)}=24.397, \mathrm{P}<0.001\right)$ on density as well as a marginally significant interaction effect $\left(F_{(8,345)}=2.278, P=0.022\right)$ when considering only superinfected individuals. For $w A l b B$, only the main effect of population was highly significant $\left(F_{(13,460)}=\right.$ $20.165, P<0.001)$ whereas the overall effect of $\operatorname{sex}\left(F_{(1,460)}=0.008, P=0.926\right)$ was not significant and there was only a marginally significant interaction $\left(F_{(10,460)}=2.028, P=\right.$ 0.029). The PNG population had the highest density of wAlbA, while Sri Lanka had the lowest. Additionally, for wAlbB, the population of Vanuatu had the highest density, while Sri Lanka again had the lowest density. 
Wolbachia density of the wAlbB infection did not differ in the singly infected and superinfected mosquitoes from Torres Strait (Fig 5A-B: $\left.F_{(1,132)}=0.399, P=0.529\right)$ and there was also no sex effect $\left(F_{(1,132)}=1.188, P 0.278\right)$ or interaction $\left(F_{(1,132)}=1.676, P=0.198\right)$. For Vietnam females, there was also no difference between the infection type in density (Fig $\left.5 C ; F_{(1,13)}=1.048, P=0.325\right)$.

There was a significant association between wAlbA and wAlbB infections in superinfected Ae. albopictus females in most populations (Fig 6A-F). Correlations between densities of the two infections were always positive and significant $(P<0.05)$ in the samples and regression analyses where the wAlbA density was used to predict the density of wAlbB were also always significant with positive slopes in each case (Fig 6). On the other hand, we found no associations between the density of the infections in males in the two populations with moderate sample sizes (>10) available for analyses (Fig 6G-H)

A

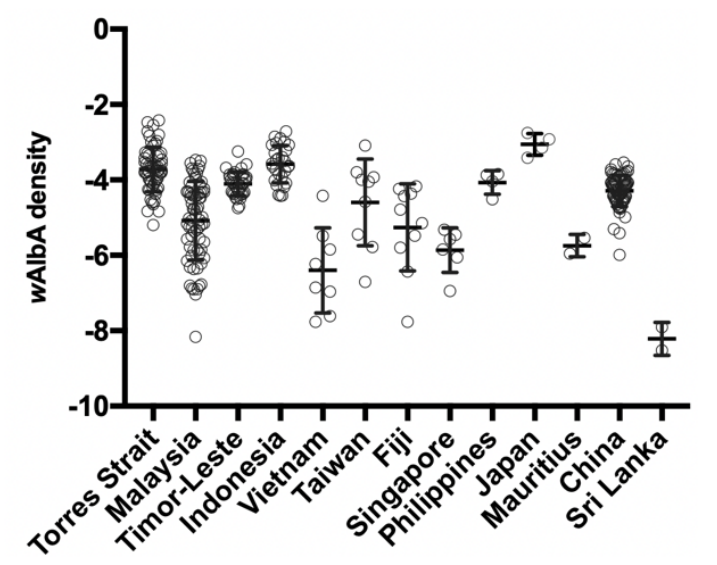

$\mathrm{B}$

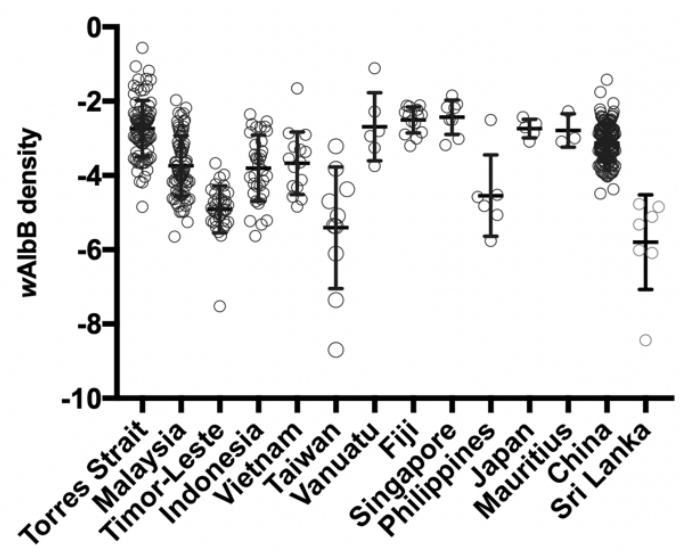

Figure 4. wAlbA density (A) and wAlbB density (B) in Aedes albopictus from each

population. Vertical lines and error bars represent medians and 95\% confidence intervals. 
Torres Strait female

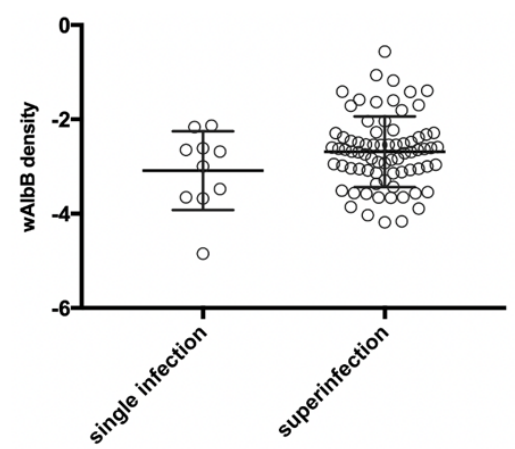

Torres Strait male

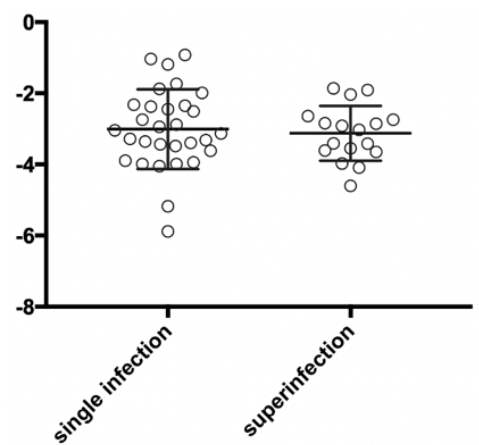

Vietnam female

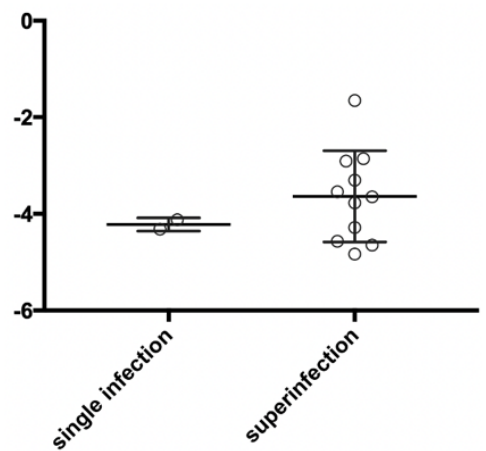

Figure 5. Female (A) and male (B) wAlbB density in single-infected and superinfected $A e$. albopictus from Torres Strait and female (C) wAlbB density in single- and superinfected Ae. albopictus from Vietnam. Vertical lines and error bars represent medians and $95 \%$ confidence intervals.

A

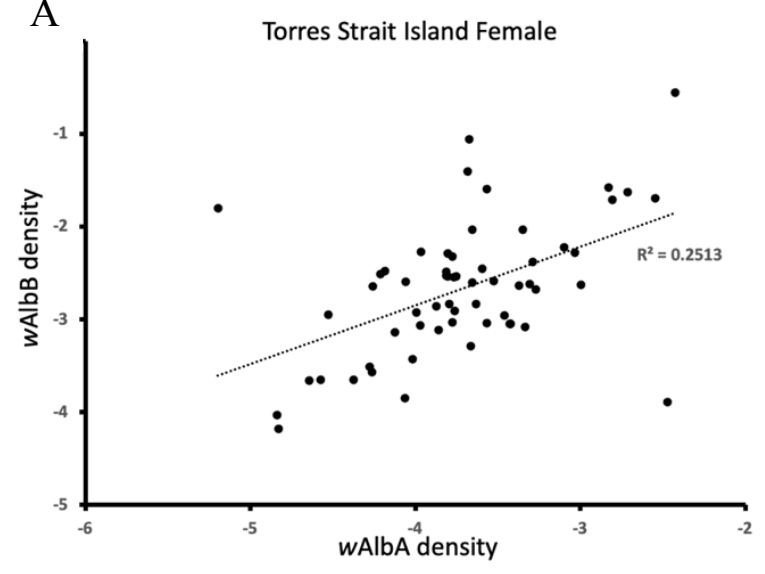

C

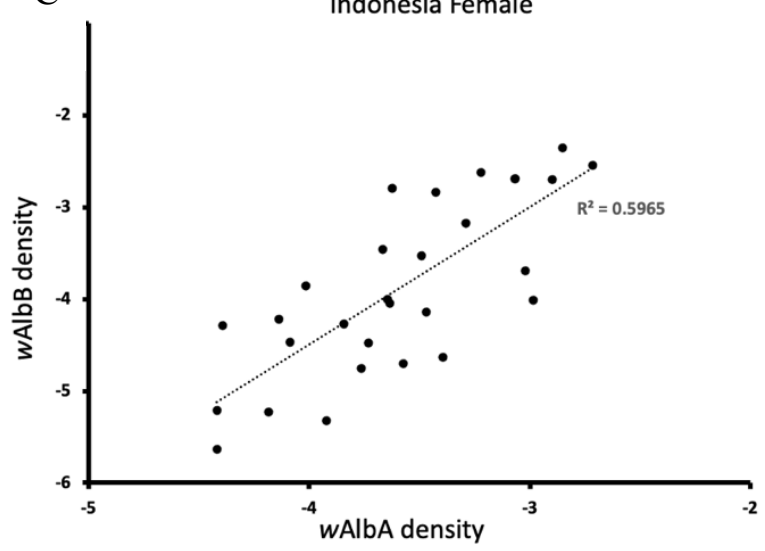

$\mathrm{E}$

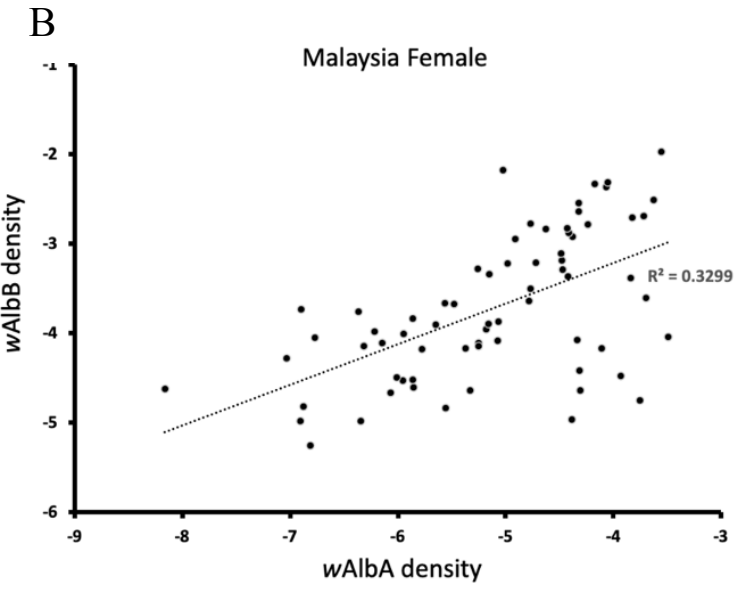

$\mathrm{D}$

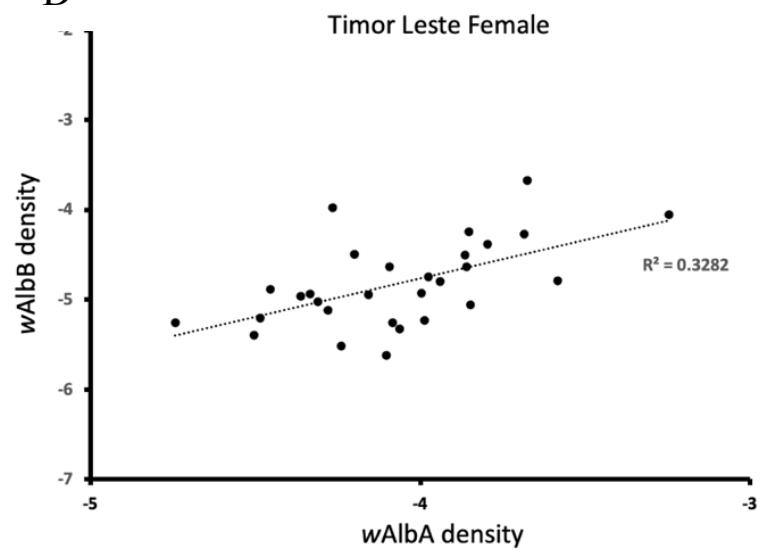

F 

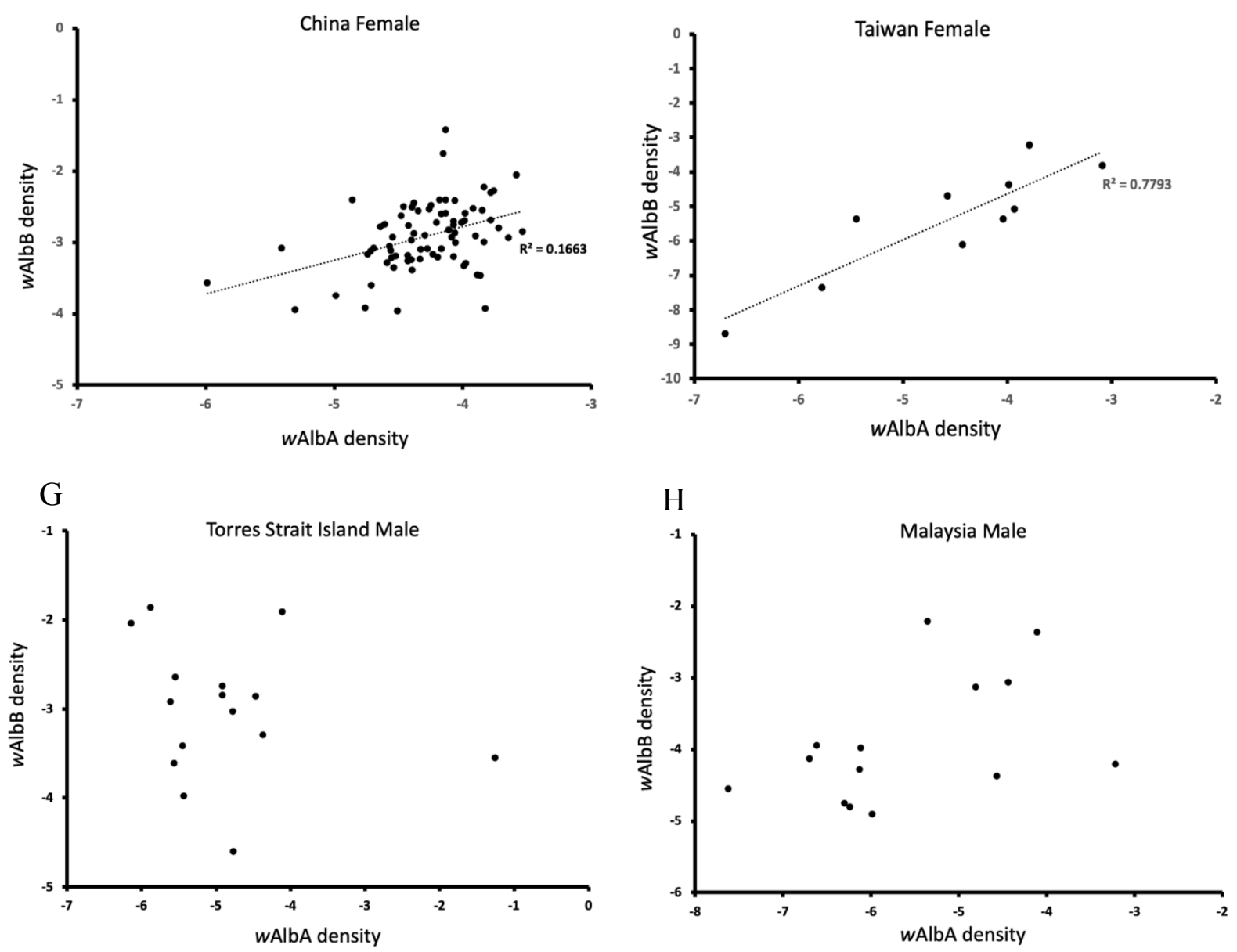

Figure 6. wAlbA density and wAlbB density in individual females (A-F) and males (G, H) across populations. Data are shown separately for samples from Torres Strait Island $(A, G)$, Malaysia $(B, H)$, Indonesia (C), Timor-Leste (D), China (E) and Taiwan (F). Regression lines are included where significant linear associations were detected.

\section{Discussion}

\section{Population history}

Overall, we found 23 mtDNA haplotypes in the Indo-Pacific samples of Ae. albopictus but no evidence of cryptic species. Minard et al. (2017) reported a novel cryptic species of Ae. albopictus in Vietnam which lacked Wolbachia [12]. Additionally, Guo et al. (2018) reported cryptic species of Ae. albopictus in China which were separated by substantial genetic distances from the other Ae. albopictus populations sampled from various regions within the country; Wolbachia was infrequent or absent in these populations of the putative cryptic species [11]. In contrast, we failed to detect the cryptic Ae. albopictus suggesting 
that they are not common across the Indo-Pacific region. This also implies that the cryptic lineage is not contributing to variation in the incidence of wAlbA and wAlbB across populations. Despite this, we found substantial variation in the incidence of Wolbachia infections, with a low incidence particularly in Timor-Leste despite the proximity of this sample to those we collected from Indonesia where both wAlbA and wAlbB infections were common.

Previous genetic analysis of Ae. albopictus populations from Asia with SNPs [15, 34] suggested three main regions of genetic similarity, one in East Asia, one in Southeast Asia (excluding Indonesia), and one in Indonesia. Some populations outside the native range showed clear signs of recent invasion from these regions; specifically, Mauritius from East Asia, and Fiji and Christmas Island from Southeast Asia [15]. A second study identified three major genetic groups: Torres Strait/Indonesia/Timor-Leste; East Asia/Southeast Asia/Fiji; and PNG/Vanuatu [34]. Gene flow from PNG into the Torres Strait was suggested by the higher co-ancestry between PNG and some Torres Strait genotypes.

Our mtDNA data confirmed three Asian range lineages of Ae. albopictus. The Indonesian lineage was highly differentiated from the others, indicating a common heritage between East Asian and Southeast Asian lineages. Shared haplotypes (H1) between PNG, Vanuatu, and Christmas Island provided the evidence of contemporaneous invasion of these populations. Shared haplotypes between Christmas Island and Sri Lanka (H9) as well as PNG, Fiji and other Southeast Asian populations ( $\mathrm{H} 11)$ grouped with all the other Southeast Asian haplotypes, suggesting recent co-ancestry between these invading populations and Southeast Asia. The presence of cryptic species in Sri Lankan populations had been previously hypothesized [15], but our phylogenetic analysis suggests there is no cryptic species within Sri Lanka populations. Nevertheless, the incongruence between the mtDNA and nuclear DNA results from the Sri Lanka samples remains of interest.

Initial genetic investigation of the Torres Strait showed that the invasion was most likely from Indonesia via a "stepping-stone" in the "Southern Fly" region of PNG [35, 36]. Our results are accord with these findings. Haplotype $2(\mathrm{H} 2)$ was found in all individuals from the Torres Strait and some individuals from the Philippines, Timor-Leste, and Indonesia, while this haplotype was not found in PNG. Considering the findings in Schmidt, Swan (34), where the Torres Strait nuclear genetic background was more like Indonesia than to Timor-Leste, 
Indonesia is supported as the most likely source of the Torres Strait invasion. $\mathrm{H} 2$ was connected to the East Asian haplogroup through a private haplotype from Timor-Leste (H8), which differed from $\mathrm{H} 2$ by one mutation step at nucleotide position 39 . These results suggest an invasion scenario in which Ae. albopictus invaded Indonesia and Timor-Leste from East Asia, and subsequently colonised the Torres Strait.

\section{Variation in Wolbachia infections across populations}

Although the wAlbA and wAlbB superinfection predominated in most populations, we did find some variation in the frequencies and densities of the two infections across populations and across sexes. Once at a high frequency, a superinfection should be maintained in a population unless transmission rates are low and host fitness costs are particularly high [19]. Invasion by a superinfection is expected to take place when the superinfection increases in frequency beyond a specific point dictated by host fitness costs, strength of $\mathrm{Cl}$ and rate of maternal transmission. Aedes albopictus females with superinfections may have higher oviposition rates and live longer [20]. However, the fitness effects of Wolbachia in Ae. albopictus appear to be complex and dependent on conditions [37] as they are in other insect systems where an infection that might appear to be deleterious can nevertheless still be beneficial in some situations [38]. Transmission of both the single infections and the superinfection appears to be close to $100 \%$ in Ae. albopictus [14] but transmission can also vary dramatically with environmental conditions as demonstrated by rearing Aedes under hot conditions [39].

Our findings reinforce the notion that there are sex differences in infection frequencies, and that the frequency of $w \mathrm{AlbB}$ as a single infection is higher in males than in females due to apparent loss of wAlbA in males [22]. Loss of an infection in the male sex is unlikely to alter the population dynamics of Wolbachia in mosquito populations that rely on transmission and selection through the female sex [40]. A single wAlbA infection can cause high levels of $\mathrm{Cl}$, allowing it to spread into an uninfected population [20,41], but ongoing selection may have led to a decrease of WAlbA in males, producing a decline of wAlbA-induced $\mathrm{CI}[20]$. Incompatible matings effectively lower the fertility of infected males, leading selection to reduce infection density in males before sexual maturation, and wild-type superinfected $A e$. 
albopictus males with very low wAlbA titres induce significantly lower levels of $\mathrm{Cl}[6]$. Superinfected females will remain compatible with males that lose the wAlbA strain as well as other males [20]. Alleles that favour loss of infection in males may therefore not necessarily be selected against in the absence of paternal transmission. In Drosophila there is also at least one $\mathrm{Cl}$ infection that is often lost in males, although in this case the males retain a reduced capacity to cause $\mathrm{Cl}$ in matings with uninfected females $[42,43]$.

Apart from sex differences, we also found substantial variation in both density and incidence of the wAlbB and wAlbA infections across populations. We can compare our results to the Wolbachia infection status of Ae. albopictus in the Indo-Pacific region determined in previous studies [11, 14, 22, 23, 44-56] (Table S2). These studies and ours highlight that the wAlbA/wAlbB superinfection or wAlbB single infection are present in most natural Ae. albopictus populations $[22,23]$. Single infections of wAlbA can occur in Ae. albopictus populations but appear variable in frequency at least in samples from Malaysia, China, and India $[11,46,53]$. Some caution is required in making comparisons between studies, given that the density of wAlbA can be low and its likelihood of detection may therefore be variable depending on the sensitivity of assays used by different laboratories. It is also possible that sample preservation following collection influences Wolbachia density and detection.

Several non-technical factors could contribute to the substantial variation in Wolbachia density across populations, including environmental conditions known to influence density such as temperature [57] and low levels of environmental antibiotics [58]. Age and life stage at collection also affect Wolbachia density $[6,22,59]$, and Wolbachia density can be affected by nuclear factors as evident from the variation in density when introduced to different host backgrounds $[60,61]$. Variation in Wolbachia genomes could also contribute to density differences, given that between-population variation exists within wAlbB $[62,63]$ and that closely related Wolbachia variants can differ in density [64].

Wolbachia infections can interact, or they can be independent in the host, but our density data provides no evidence of competition between the two Wolbachia strains. Although there was a lower density of WAlbA in mosquitoes as has previously been noted $[6,65]$, we found no evidence that the presence or density of WAlbA had a negative effect on the density of $W A \mathrm{AlbB}$ and vice versa. In superinfected mosquitoes, there was a consistent 
positive association of these infections in females from all samples and an absence of any pattern in males, highlighting the absence of antagonistic interactions among the infections.

\section{Strain characterization via SNPS}

Our sexing data shows the usefulness of SNPs not only in population studies but also as markers that can be repeatedly used to investigate new issues as they arise. SNP markers have previously provided information on population structure and history across the IndoPacific region [15] and provided information on quarantine risk identification [66]. We also applied these markers to dissect patterns of selection on pesticide resistance markers [67] and have now used them to identify genomic features that have their sequencing depths associated with sex. With sufficient sequencing depth, RAD sequencing is a viable way to determine the sex of Ae. albopictus. Whereas sexing using morphology can be time consuming and impossible for immature samples and poorly preserved material, sex classification using ML can be automated as part of the processing workflow when working with RAD-seq data.

While SNP-based sexing worked well, we found a moderate amount of disagreement between the Wolbachia infection status from the SNP-based ML model and the status given from the qPCR assay. There are several possible explanations for this. First, the SNP features chosen for the ML model may not truly be able to separate the two Wolbachia strains. Second, cross-sample contamination of DNA may have led to the presence of wAlbA or wAlbB sequencing reads in non-infected samples. Lastly, extremely low density of Wolbachia may lead to screening inaccuracies when densities fall under the limit of detection by qPCR $[68,69]$.

The results from the strain classification model were also heavily influenced by the relative proportions of classes in the training data. Due to being trained on a dataset where the number of superinfected individuals was approximately six times greater than the number of single wAlbB individuals, a sample with a low number of allelic observations, either due to low sequencing depth or low Wolbachia density, is more likely to be classified as superinfected than singly infected by wAlbA. Additionally, since there were insufficient singly infected wAlbA samples to train the model to classify singly infected wAlbA cases, new data with wAlbA would likely be classified as superinfected. Therefore, when classifying 
future samples from different populations, thus having different priors of $w A l b A / w A l b B$ proportions, the existing model should perhaps be discarded, and the relative numbers of observed alleles from wAlbA/wAlbB could instead be used as a heuristic to classify samples.

\section{Applied implications}

In the absence of cryptic species of Ae. albopictus and given the substantial variation in infection frequency, we suspect that Wolbachia-based interventions targeting this species are applicable across the Indo-Pacific region. Strains generated through transinfection and utilised in one location [70] may therefore be suitable for other regions as long as the nuclear background of the strain is altered to match that of the target population in case of local adaptation and local pesticide resistance levels [71]. However, given the preponderance of superinfected mosquitoes, it will be important to release individuals with new infections that are capable of invading local populations or supressing them via $\mathrm{Cl}$. We also emphasize the usefulness of SNPs in applied population studies more generally and as markers that can be repeatedly reanalysed to investigate new issues as these arise.

\section{References}

1. Gratz NG. Critical review of the vector status of Aedes albopictus. Med Vet Entomol. 2004;18(3):215-27. Epub 2004/09/07. doi: 10.1111/j.0269-283X.2004.00513.x. PubMed PMID: 15347388.

2. Bhatt S, Gething PW, Brady OJ, Messina JP, Farlow AW, Moyes CL, et al. The global distribution and burden of dengue. Nature. 2013;496(7446):504-7. Epub 2013/04/09. doi: 10.1038/nature12060. PubMed PMID: 23563266; PubMed Central PMCID: PMCPMC3651993.

3. Vontas J, Kioulosb E, Pavlidia N, Moroua E, Torrect A, Ransoncd H. Insecticide resistance in the major dengue vectors Aedes albopictus and Aedes aegypti. Pestic Biochem Physiol. 2012;104(2):126-31.

4. Demok S, Endersby-Harshman N, Vinit R, Timinao L, Robins L, Susapu M, et al. Insecticide resistance status of Aedes aegypti and Aedes albopictus mosquitoes in Papua New Guinea. Parasit Vectors. 2019;12(1).

5. Fikrig K, Harrington LC. Understanding and interpreting mosquito blood feeding studies: the case of Aedes albopictus. Trends Parasitol. 2021;37(11):959-75. Epub 2021/09/10. doi: 10.1016/j.pt.2021.07.013. PubMed PMID: 34497032.

6. Calvitti M, Marini F, Desiderio A, Puggioli A, Moretti R. Wolbachia density and cytoplasmic incompatibility in Aedes albopictus: concerns with using artificial Wolbachia infection as a vector suppression tool. PLoS One. 2015;10(3):e0121813. Epub 2015/03/27. doi:

10.1371/journal.pone.0121813. PubMed PMID: 25812130; PubMed Central PMCID: PMCPMC4374832.

7. Xi Z, Dean JL, Khoo C, Dobson SL. Generation of a novel Wolbachia infection in Aedes albopictus (Asian tiger mosquito) via embryonic microinjection. Insect Biochem Mol Biol. 2005;35(8):903-10. Epub 2005/06/10. doi: 10.1016/j.ibmb.2005.03.015. PubMed PMID: 15944085; PubMed Central PMCID: PMCPMC1410910. 
8. Zheng X, Zhang D, Li Y, Yang C, Wu Y, Liang X, et al. Incompatible and sterile insect techniques combined eliminate mosquitoes. Nature. 2019;572(7767):56-61. Epub 2019/07/19. doi: 10.1038/s41586-019-1407-9. PubMed PMID: 31316207.

9. Blagrove MS, Arias-Goeta C, Failloux AB, Sinkins SP. Wolbachia strain wMel induces cytoplasmic incompatibility and blocks dengue transmission in Aedes albopictus. Proc Natl Acad Sci U S A. 2012;109(1):255-60. Epub 2011/11/30. doi: 10.1073/pnas.1112021108. PubMed PMID: 22123944; PubMed Central PMCID: PMCPMC3252941.

10. Moretti R, Yen P-S, Houé V, Lampazzi E, Desiderio A, Failloux A-B, et al. Combining Wolbachia-induced sterility and virus protection to fight Aedes albopictus-borne viruses. PLoS neglected tropical diseases. 2018;12(7):e0006626.

11. Guo Y, Song Z, Luo L, Wang Q, Zhou G, Yang D, et al. Molecular evidence for new sympatric cryptic species of Aedes albopictus (Diptera: Culicidae) in China: A new threat from Aedes albopictus subgroup? Parasit Vectors. 2018;11(1):228. Epub 2018/04/06. doi: 10.1186/s13071-018-2814-8. PubMed PMID: 29618379; PubMed Central PMCID: PMCPMC5885320.

12. Minard G, Tran Van V, Tran FH, Melaun C, Klimpel S, Koch LK, et al. Identification of sympatric cryptic species of Aedes albopictus subgroup in Vietnam: new perspectives in phylosymbiosis of insect vector. Parasit Vectors. 2017;10(1):276. Epub 2017/06/05. doi: 10.1186/s13071-017-2202-9. PubMed PMID: 28577575; PubMed Central PMCID: PMCPMC5457575. 13. Sinkins SP, Braig HR, O'Neill SL. Wolbachia superinfections and the expression of cytoplasmic incompatibility. Proc Biol Sci. 1995;261(1362):325-30. Epub 1995/09/22. doi:

10.1098/rspb.1995.0154. PubMed PMID: 8587875.

14. Kittayapong P, Baisley KJ, Sharpe RG, Baimai V, O'Neill SL. Maternal transmission efficiency of Wolbachia superinfections in Aedes albopictus populations in Thailand. The American journal of tropical medicine and hygiene. 2002;66(1):103-7.

15. Schmidt TL, Chung J, Honnen AC, Weeks AR, Hoffmann AA. Population genomics of two invasive mosquitoes (Aedes aegypti and Aedes albopictus) from the Indo-Pacific. PLoS NegI Trop Dis. 2020;14(7):e0008463. Epub 2020/07/18. doi: 10.1371/journal.pntd.0008463. PubMed PMID: 32678817; PubMed Central PMCID: PMCPMC7390453.

16. Medley KA, Westby KM, Jenkins DG. Rapid local adaptation to northern winters in the invasive Asian tiger mosquito Aedes albopictus: A moving target. J Appl Ecol. 2019;56(11):2518-27. 17. Ross PA, Turelli M, Hoffmann AA. Evolutionary Ecology of Wolbachia Releases for Disease Control. Annu Rev Genet. 2019;53:93-116. Epub 2019/09/11. doi: 10.1146/annurev-genet-112618043609. PubMed PMID: 31505135; PubMed Central PMCID: PMCPMC6944334.

18. Dobson SL, Rattanadechakul W, Marsland EJ. Fitness advantage and cytoplasmic incompatibility in Wolbachia single- and superinfected Aedes albopictus. Heredity (Edinb). 2004;93(2):135-42. Epub 2004/05/06. doi: 10.1038/sj.hdy.6800458. PubMed PMID: 15127087. 19. Hoffmann AA, Turelli M. Cytoplasmic incompatibility in insects. Influential Passengers: Inherited Microorganisms and Invertebrate Reproduction. 1997:42-80.

20. Dobson SL, Marsland EJ, Rattanadechakul W. Wolbachia-induced cytoplasmic incompatibility in single- and superinfected Aedes albopictus (Diptera: Culicidae). J Med Entomol. 2001;38(3):382-7. Epub 2001/05/25. doi: 10.1603/0022-2585-38.3.382. PubMed PMID: 11372962.

21. Ross PA, Ritchie SA, Axford JK, Hoffmann AA. Loss of cytoplasmic incompatibility in Wolbachia-infected Aedes aegypti under field conditions. PLoS Negl Trop Dis. 2019;13(4):e0007357. Epub 2019/04/20. doi: 10.1371/journal.pntd.0007357. PubMed PMID: 31002720; PubMed Central PMCID: PMCPMC6493766.

22. Tortosa P, Charlat S, Labbe P, Dehecq JS, Barre H, Weill M. Wolbachia age-sex-specific density in Aedes albopictus: a host evolutionary response to cytoplasmic incompatibility? PLoS One. 2010;5(3):e9700. Epub 2010/03/20. doi: 10.1371/journal.pone.0009700. PubMed PMID: 20300514; PubMed Central PMCID: PMCPMC2838780. 
23. Afizah AN, Mahirah MN, Azahari AH, Asuad MK, Nazni WA, Lee HL. Absence of Aedes Aegypti (L.) on an Ecological Island: Competitive Exclusion? Southeast Asian J Trop Med Public Health. 2015;46(5):850-6. Epub 2016/02/13. PubMed PMID: 26863856.

24. Fontaine A, Filipovic I, Fansiri T, Hoffmann AA, Cheng C, Kirkpatrick M, et al. Extensive Genetic Differentiation between Homomorphic Sex Chromosomes in the Mosquito Vector, Aedes aegypti. Genome Biol Evol. 2017;9(9):2322-35. Epub 2017/09/26. doi: 10.1093/gbe/evx171. PubMed PMID: 28945882; PubMed Central PMCID: PMCPMC5737474.

25. Oliver KM, Moran NA, Hunter MS. Costs and benefits of a superinfection of facultative symbionts in aphids. Proc Biol Sci. 2006;273(1591):1273-80. Epub 2006/05/25. doi: 10.1098/rspb.2005.3436. PubMed PMID: 16720402; PubMed Central PMCID: PMCPMC1560284. 26. Folmer $\mathrm{O}$, Black $\mathrm{M}$, Hoeh $\mathrm{W}$, Lutz $\mathrm{R}$, Vrijenhoek R. DNA primers for amplification of mitochondrial cytochrome $\mathrm{c}$ oxidase subunit I from diverse metazoan invertebrates. Mol Mar Biol Biotechnol. 1994;3(5):294-9. Epub 1994/10/01. PubMed PMID: 7881515.

27. Li H. Aligning sequence reads, clone sequences and assembly contigs with BWA-MEM. arXiv:13033997v2 [q-bioGN]. 2013.

28. Meyer D, Dimitriadou E, Hornik K, Weingessel A, Leisch F, Chang CC, et al. e1071: Misc functions of the Department of Statistics (e1071), TU Wien. R package version. 2014;1(3).

29. Danecek P, Bonfield JK, Liddle J, Marshall J, Ohan V, Pollard MO, et al. Twelve years of SAMtools and BCFtools. Gigascience. 2021;10(2). Epub 2021/02/17. doi:

10.1093/gigascience/giab008. PubMed PMID: 33590861; PubMed Central PMCID: PMCPMC7931819.

30. Langmead B, Salzberg SL. Fast gapped-read alignment with Bowtie 2. Nat Methods. 2012;9(4):357-9. Epub 2012/03/06. doi: 10.1038/nmeth.1923. PubMed PMID: 22388286; PubMed Central PMCID: PMCPMC3322381.

31. Garrison E, Marth G. Haplotype-based variant detection from short-read sequencing. arXiv preprint arXiv:12073907 [q-bioGN]. 2012.

32. Lee SF, White VL, Weeks AR, Hoffmann AA, Endersby NM. High-throughput PCR assays to monitor Wolbachia infection in the dengue mosquito (Aedes aegypti) and Drosophila simulans. Appl Environ Microbiol. 2012;78(13):4740-3. Epub 2012/04/24. doi: 10.1128/AEM.00069-12. PubMed PMID: 22522691; PubMed Central PMCID: PMCPMC3370494.

33. Joubert DA, Walker T, Carrington LB, De Bruyne JT, Kien DH, Hoang Nle T, et al. Establishment of a Wolbachia Superinfection in Aedes aegypti Mosquitoes as a Potential Approach for Future Resistance Management. PLoS Pathog. 2016;12(2):e1005434. Epub 2016/02/20. doi: 10.1371/journal.ppat.1005434. PubMed PMID: 26891349; PubMed Central PMCID: PMCPMC4758728.

34. Schmidt TL, Swan T, Chung J, Karl S, Demok S, Yang Q, et al. Spatial population genomics of a recent mosquito invasion. Mol Ecol. 2021;30(5):1174-89. Epub 2021/01/10. doi:

10.1111/mec.15792. PubMed PMID: 33421231.

35. Beebe NW, Ambrose L, Hill LA, Davis JB, Hapgood G, Cooper RD, et al. Tracing the tiger: population genetics provides valuable insights into the Aedes (Stegomyia) albopictus invasion of the Australasian Region. PLoS Negl Trop Dis. 2013;7(8):e2361. Epub 2013/08/21. doi:

10.1371/journal.pntd.0002361. PubMed PMID: 23951380; PubMed Central PMCID: PMCPMC3738475.

36. Maynard AJ, Ambrose L, Cooper RD, Chow WK, Davis JB, Muzari MO, et al. Tiger on the prowl: Invasion history and spatio-temporal genetic structure of the Asian tiger mosquito Aedes albopictus (Skuse 1894) in the Indo-Pacific. PLoS Negl Trop Dis. 2017;11(4):e0005546. Epub 2017/04/15. doi: 10.1371/journal.pntd.0005546. PubMed PMID: 28410388; PubMed Central PMCID: PMCPMC5406021.

37. Gavotte L, Mercer DR, Stoeckle JJ, Dobson SL. Costs and benefits of Wolbachia infection in immature Aedes albopictus depend upon sex and competition level. Journal of invertebrate pathology. 2010;105(3):341-6. 
38. Yeap HL, Mee P, Walker T, Weeks AR, O'Neill SL, Johnson P, et al. Dynamics of the "popcorn" Wolbachia infection in outbred Aedes aegypti informs prospects for mosquito vector control. Genetics. 2011;187(2):583-95. Epub 2010/12/08. doi: 10.1534/genetics.110.122390. PubMed PMID: 21135075; PubMed Central PMCID: PMCPMC3030498.

39. Ross PA, Wiwatanaratanabutr I, Axford JK, White VL, Endersby-Harshman NM, Hoffmann AA. Wolbachia Infections in Aedes aegypti Differ Markedly in Their Response to Cyclical Heat Stress. Plos Pathogens. 2017;13(1). doi: ARTN e100600610.1371/journal.ppat.1006006. PubMed PMID: WOS:000395743500002.

40. Sinkins SP. Wolbachia and cytoplasmic incompatibility in mosquitoes. Insect Biochem Mol Biol. 2004;34(7):723-9. Epub 2004/07/10. doi: 10.1016/j.ibmb.2004.03.025. PubMed PMID: 15242714.

41. Dutton TJ, Sinkins SP. Strain-specific quantification of Wolbachia density in Aedes albopictus and effects of larval rearing conditions. Insect Mol Biol. 2004;13(3):317-22. Epub 2004/05/26. doi: 10.1111/j.0962-1075.2004.00490.x. PubMed PMID: 15157232.

42. Richardson KM, Griffin PC, Lee SF, Ross PA, Endersby-Harshman NM, Schiffer M, et al. A Wolbachia infection from Drosophila that causes cytoplasmic incompatibility despite low prevalence and densities in males. Heredity (Edinb). 2019;122(4):428-40. Epub 2018/08/25. doi:

10.1038/s41437-018-0133-7. PubMed PMID: 30139962; PubMed Central PMCID: PMCPMC6460763. 43. Hoffmann AA, Turelli M, Harshman LG. Factors affecting the distribution of cytoplasmic incompatibility in Drosophila simulans. Genetics. 1990;126(4):933-48. Epub 1990/12/01. doi: 10.1093/genetics/126.4.933. PubMed PMID: 2076821; PubMed Central PMCID: PMCPMC1204290. 44. Ahmad NA, Vythilingam I, Lim YAL, Zabari N, Lee HL. Detection of Wolbachia in Aedes albopictus and Their Effects on Chikungunya Virus. Am J Trop Med Hyg. 2017;96(1):148-56. Epub 2016/12/07. doi: 10.4269/ajtmh.16-0516. PubMed PMID: 27920393; PubMed Central PMCID: PMCPMC5239683.

45. Joanne S, Vythilingam I, Yugavathy N, Leong CS, Wong ML, AbuBakar S. Distribution and dynamics of Wolbachia infection in Malaysian Aedes albopictus. Acta Trop. 2015;148:38-45. Epub 2015/04/23. doi: 10.1016/j.actatropica.2015.04.003. PubMed PMID: 25899523.

46. Noor-Shazleen-Husnie MM, Emelia O, Ahmad-Firdaus MS, Zainol-Ariffin P, Aishah-Hani A. Detection of Wolbachia in wild mosquito populations from selected areas in Peninsular Malaysia by loop-mediated isothermal amplification (LAMP) technique. Trop Biomed. 2018;35(2):330-46. Epub 2018/06/01. PubMed PMID: 33601807.

47. Park CH, Lim H, Kim H, Lee WG, Roh JY, Park MY, et al. High prevalence of Wolbachia infection in Korean populations of Aedes albopictus (Diptera: Culicidae). Journal of Asia-Pacific Entomology. 2016;19:191-4.

48. Shaikevich EV, Patraman IV, Bogacheva AS, Rakova VM, Zelya OP, Ganushkina LA. Invasive mosquito species Aedes albopictus and Aedes aegypti on the Black Sea coast of the Caucasus: genetics (COI, ITS2), Wolbachia and Dirofilaria infections. Vavilov Journal of Genetics and Breeding. 2018;22(5):574-85. doi: 10.18699/VJ18.397.

49. Wu YN, Wen S, Liang Qg, Yang X, Wu JH, Zhu RF, et al. The Preliminary Investigation on Symbiotic Bacteria (Wolbachia) of Common Mosquito in Guiyang. Journal of Guizhou Medical University. 2017;10:1130-3.

50. Zhang D, Zhan X, Wu X, Yang X, Liang G, Zheng Z, et al. A field survey for Wolbchia and phage WO infections of Aedes albopictus in Guangzhou City, China. Parasitol Res. 2014;113(1):399-404. Epub 2013/11/14. doi: 10.1007/s00436-013-3668-9. PubMed PMID: 24221888.

51. Chuchuy A, Rodriguero MS, Ferrari W, Ciota AT, Kramer LD, Micieli MV. Biological characterization of Aedes albopictus (Diptera: Culicidae) in Argentina: implications for arbovirus transmission. Sci Rep. 2018;8(1):5041. Epub 2018/03/24. doi: 10.1038/s41598-018-23401-7. PubMed PMID: 29568046; PubMed Central PMCID: PMCPMC5864732.

52. Armbruster P, Damsky WE, Jr., Giordano R, Birungi J, Munstermann LE, Conn JE. Infection of New- and Old-World Aedes albopictus (Diptera: Culicidae) by the intracellular parasite Wolbachia: 
implications for host mitochondrial DNA evolution. J Med Entomol. 2003;40(3):356-60. Epub 2003/08/29. doi: 10.1603/0022-2585-40.3.356. PubMed PMID: 12943116.

53. Das B, Satapathy T, Kar SK, Hazra RK. Genetic structure and Wolbachia genotyping in naturally occurring populations of Aedes albopictus across contiguous landscapes of Orissa, India. PLoS One. 2014;9(4):e94094. Epub 2014/04/10. doi: 10.1371/journal.pone.0094094. PubMed PMID: 24714653; PubMed Central PMCID: PMCPMC3979767.

54. Zouache K, Raharimalala FN, Raquin V, Tran-Van V, Raveloson LH, Ravelonandro P, et al. Bacterial diversity of field-caught mosquitoes, Aedes albopictus and Aedes aegypti, from different geographic regions of Madagascar. FEMS Microbiol Ecol. 2011;75(3):377-89. Epub 2010/12/24. doi: 10.1111/j.1574-6941.2010.01012.x. PubMed PMID: 21175696.

55. Kulkarni A, Yu W, Jiang J, Sanchez C, Karna AK, Martinez KJL, et al. Wolbachia pipientis occurs in Aedes aegypti populations in New Mexico and Florida, USA. Ecol Evol. 2019;9(10):6148-56. Epub 2019/06/05. doi: 10.1002/ece3.5198. PubMed PMID: 31161026; PubMed Central PMCID: PMCPMC6540660.

56. de Albuquerque AL, Magalhaes T, Ayres CF. High prevalence and lack of diversity of Wolbachia pipientis in Aedes albopictus populations from Northeast Brazil. Mem Inst Oswaldo Cruz. 2011;106(6):773-6. Epub 2011/10/21. doi: 10.1590/s0074-02762011000600021. PubMed PMID: 22012236.

57. Ross PA, Axford JK, Yang Q, Staunton KM, Ritchie SA, Richardson KM, et al. Heatwaves cause fluctuations in wMel Wolbachia densities and frequencies in Aedes aegypti. PLoS Negl Trop Dis. 2020;14(1):e0007958. Epub 2020/01/24. doi: 10.1371/journal.pntd.0007958. PubMed PMID: 31971938; PubMed Central PMCID: PMCPMC6977724.

58. Endersby-Harshman NM, Axford JK, Hoffmann AA. Environmental Concentrations of Antibiotics May Diminish Wolbachia infections in Aedes aegypti (Diptera: Culicidae). Journal of Medical Entomology. 2019;56(4):1078-86. doi: 10.1093/jme/tjz023.

59. Wiwatanaratanabutr I, Kittayapong P. Effects of crowding and temperature on Wolbachia infection density among life cycle stages of Aedes albopictus. J Invertebr Pathol. 2009;102(3):220-4. Epub 2009/08/19. doi: 10.1016/j.jip.2009.08.009. PubMed PMID: 19686755.

60. Kondo N, Shimada M, Fukatsu T. Infection density of Wolbachia endosymbiont affected by co-infection and host genotype. Biol Lett. 2005;1(4):488-91. Epub 2006/12/07. doi: 10.1098/rsbl.2005.0340. PubMed PMID: 17148240; PubMed Central PMCID: PMCPMC1626364.

61. Mouton L, Henri H, Charif D, Bouletreau M, Vavre F. Interaction between host genotype and environmental conditions affects bacterial density in Wolbachia symbiosis. Biol Lett. 2007;3(2):2103. Epub 2007/01/26. doi: 10.1098/rsbl.2006.0590. PubMed PMID: 17251124; PubMed Central PMCID: PMCPMC2375926.

62. Scholz M, Albanese D, Tuohy K, Donati C, Segata N, Rota-Stabelli O. Large scale genome reconstructions illuminate Wolbachia evolution. Nat Commun. 2020;11(1):5235. Epub 2020/10/18. doi: 10.1038/s41467-020-19016-0. PubMed PMID: 33067437; PubMed Central PMCID: PMCPMC7568565.

63. Ross PA, Gu X, Robinson KL, Yang Q, Cottingham E, Zhang Y, et al. A wAlbB Wolbachia transinfection displays stable phenotypic effects across divergent Aedes aegypti mosquito backgrounds. Appl Environ Microbiol. 2021;87(20):e0126421. Epub 2021/08/12. doi: 10.1128/AEM.01264-21. PubMed PMID: 34379518; PubMed Central PMCID: PMCPMC8478461. 64. Chrostek E, Marialva MS, Esteves SS, Weinert LA, Martinez J, Jiggins FM, et al. Wolbachia variants induce differential protection to viruses in Drosophila melanogaster: a phenotypic and phylogenomic analysis. PLoS Genet. 2013;9(12):e1003896. Epub 2013/12/19. doi: 10.1371/journal.pgen.1003896. PubMed PMID: 24348259; PubMed Central PMCID: PMCPMC3861217.

65. Dutton TJ, Sinkins SP. Strain-specific quantification of Wolbachia density in Aedes albopictus and effects of larval rearing conditions. Insect molecular biology. 2004;13(3):317-22. 
66. Schmidt TL, Chung J, van Rooyen AR, Sly A, Weeks AR, Hoffmann AA. Incursion pathways of the Asian tiger mosquito (Aedes albopictus) into Australia contrast sharply with those of the yellow fever mosquito (Aedes aegypti). Pest Manag Sci. 2020;76(12):4202-9. Epub 2020/06/28. doi: 10.1002/ps.5977. PubMed PMID: 32592440.

67. Endersby-Harshman NM, Schmidt TL, Chung J, van Rooyen A, Weeks AR, Hoffmann AA. Heterogeneous genetic invasions of three insecticide resistance mutations in Indo-Pacific populations of Aedes aegypti (L.). Mol Ecol. 2020;29(9):1628-41. Epub 2020/04/05. doi: 10.1111/mec.15430. PubMed PMID: 32246542.

68. Rakotonirina A, Caruzzo C, Ballan V, Kainiu M, Marin M, Colot J, et al. Wolbachia detection in Aedes aegypti using MALDI-TOF MS coupled to artificial intelligence. Sci Rep. 2021;11(1):21355. Epub 2021/11/03. doi: 10.1038/s41598-021-00888-1. PubMed PMID: 34725401; PubMed Central PMCID: PMCPMC8560810.

69. Turba ME, Zambon E, Zannoni A, Russo S, Gentilini F. Detection of Wolbachia DNA in blood for diagnosing filaria-associated syndromes in cats. J Clin Microbiol. 2012;50(8):2624-30. Epub 2012/06/01. doi: 10.1128/JCM.00528-12. PubMed PMID: 22649020; PubMed Central PMCID: PMCPMC3421487.

70. Zhang D, Lees RS, Xi Z, Bourtzis K, Gilles JR. Combining the Sterile Insect Technique with the Incompatible Insect Technique: III-Robust Mating Competitiveness of Irradiated Triple WolbachiaInfected Aedes albopictus Males under Semi-Field Conditions. PLoS One. 2016;11(3):e0151864. Epub 2016/03/19. doi: 10.1371/journal.pone.0151864. PubMed PMID: 26990981; PubMed Central PMCID: PMCPMC4798476.

71. Garcia GA, Sylvestre G, Aguiar R, da Costa GB, Martins AJ, Lima JBP, et al. Matching the genetics of released and local Aedes aegypti populations is critical to assure Wolbachia invasion. PLoS Negl Trop Dis. 2019;13(1):e0007023. Epub 2019/01/09. doi: 10.1371/journal.pntd.0007023. PubMed PMID: 30620733; PubMed Central PMCID: PMCPMC6338382. 


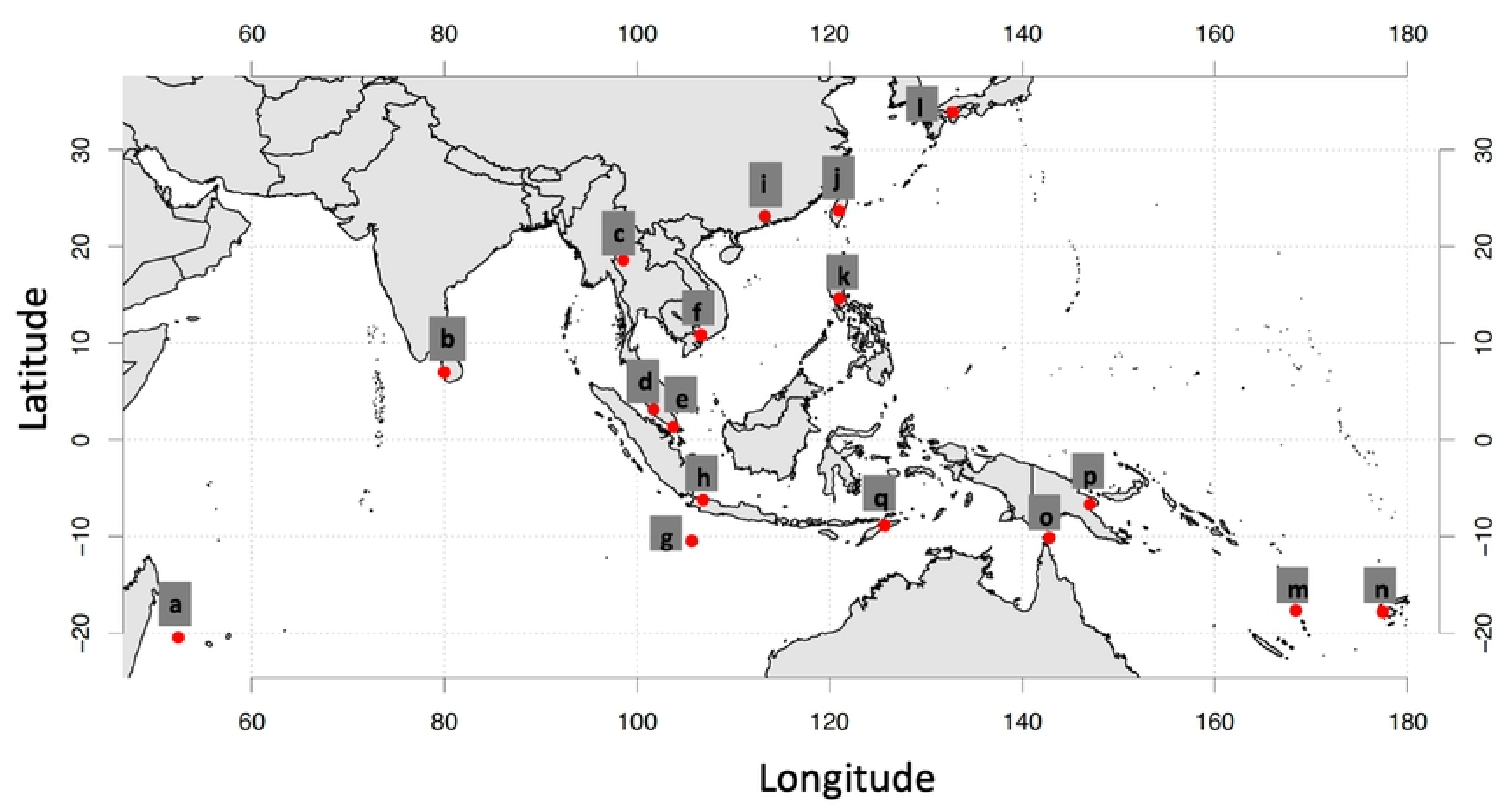

Fig1 



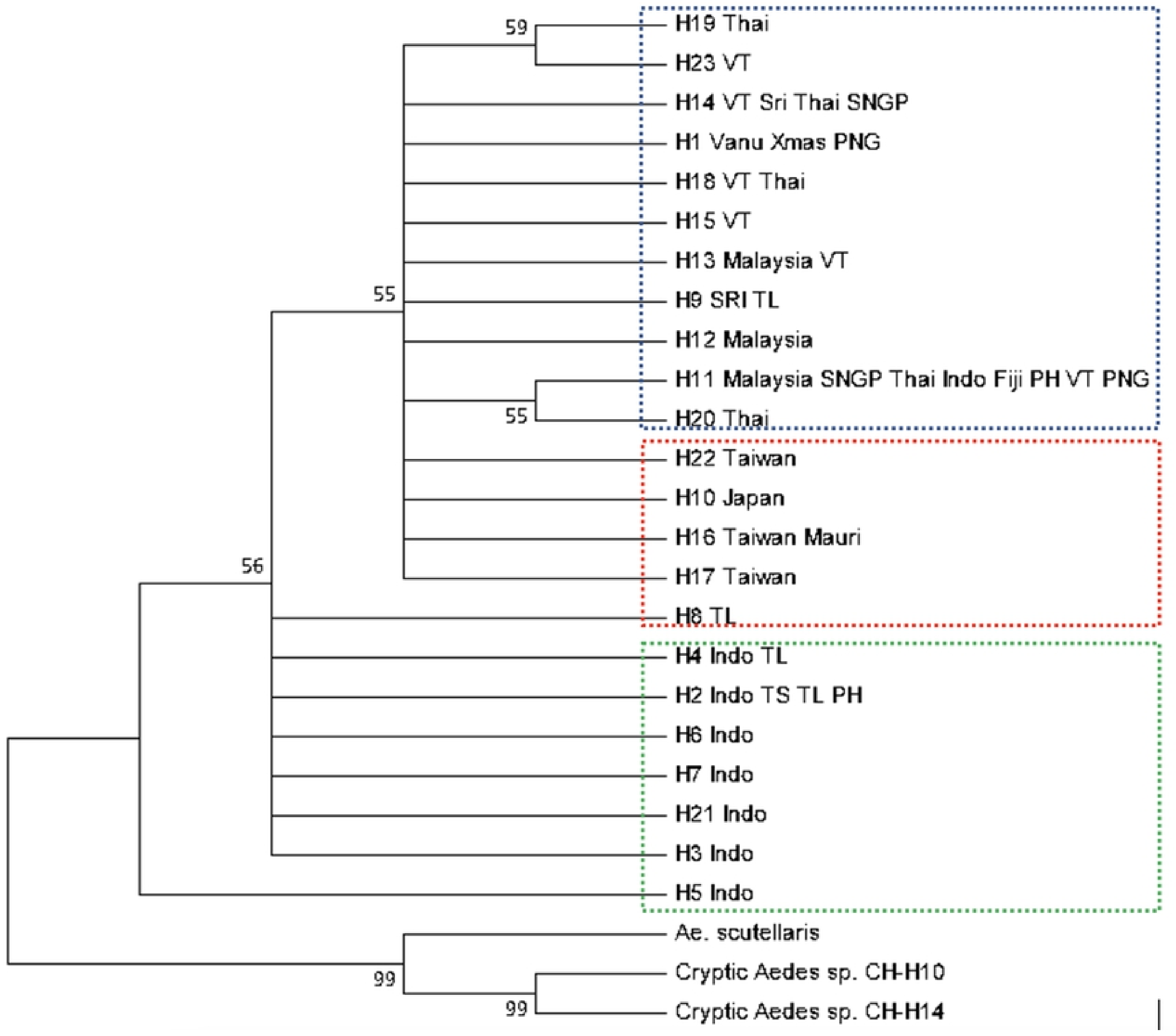

Fig3 


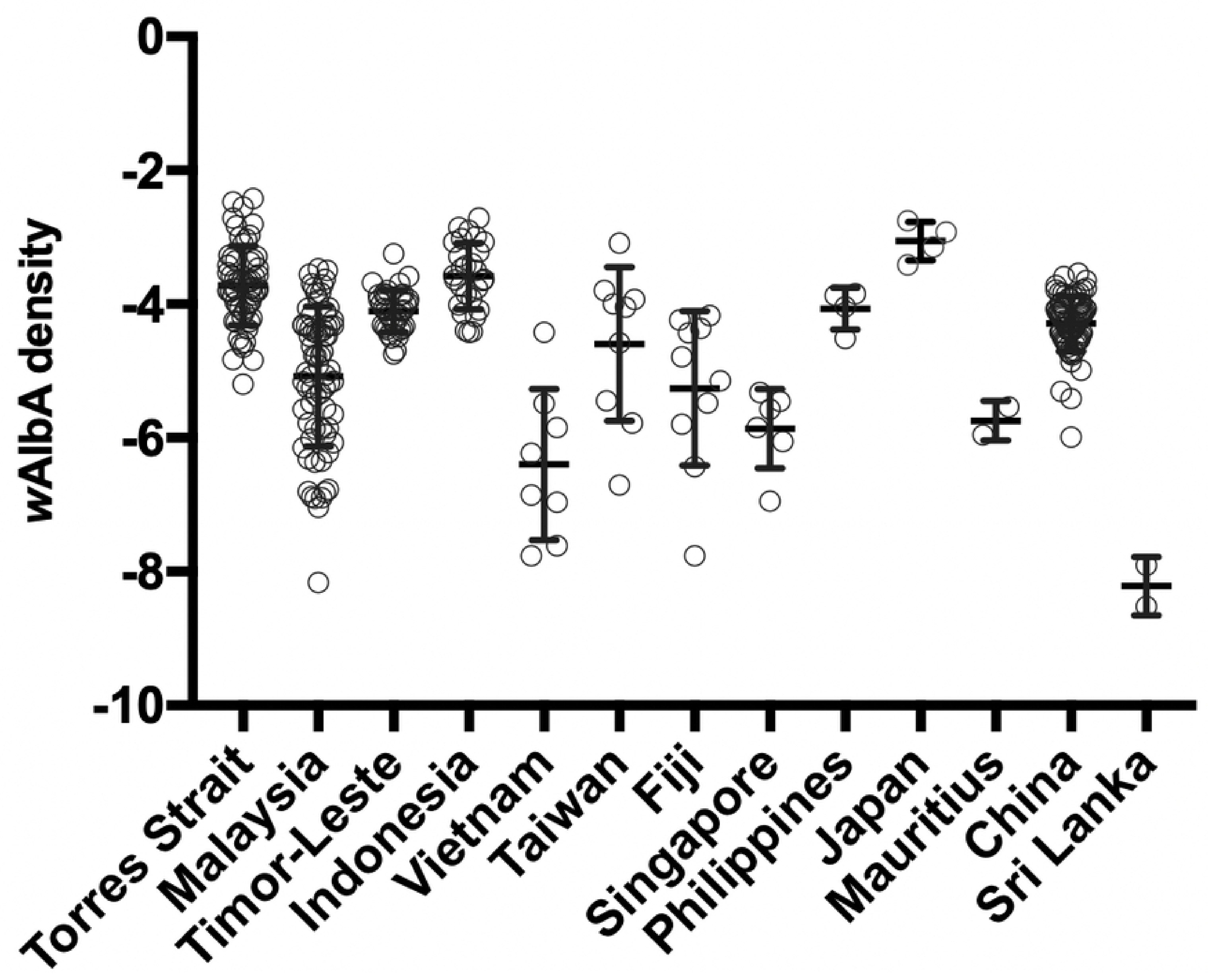

Fig4A 


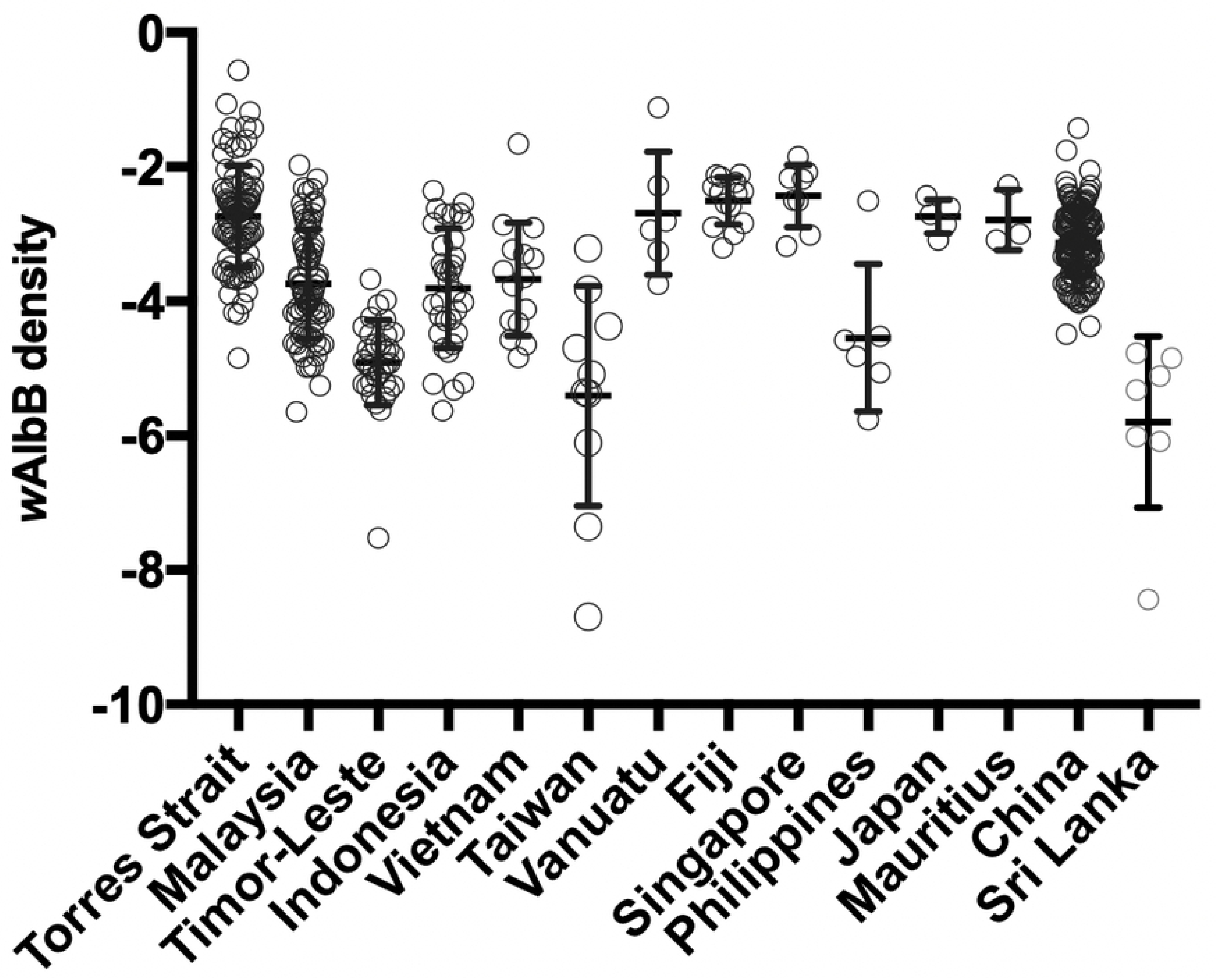

Fig4B 


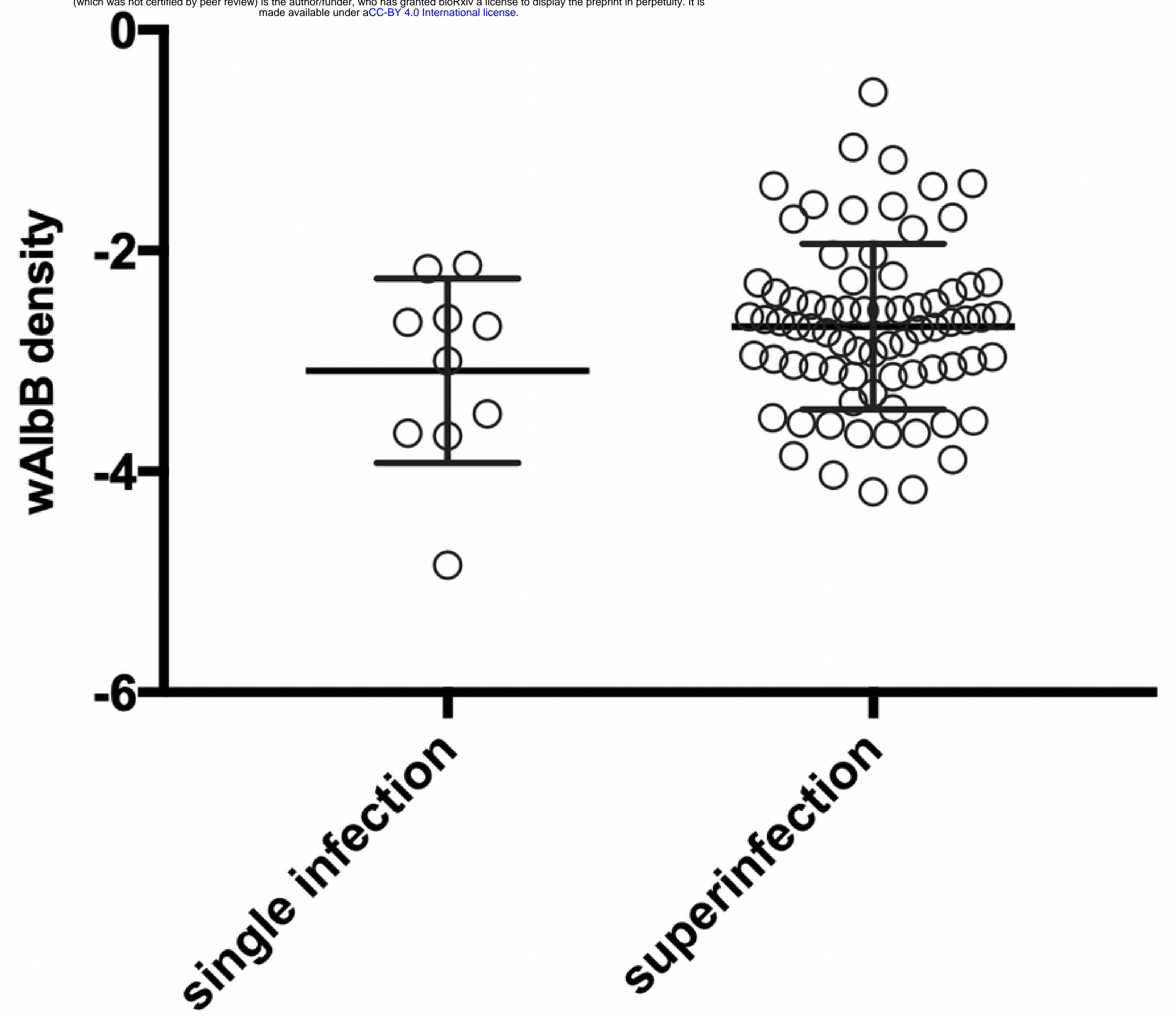

Fig5A 


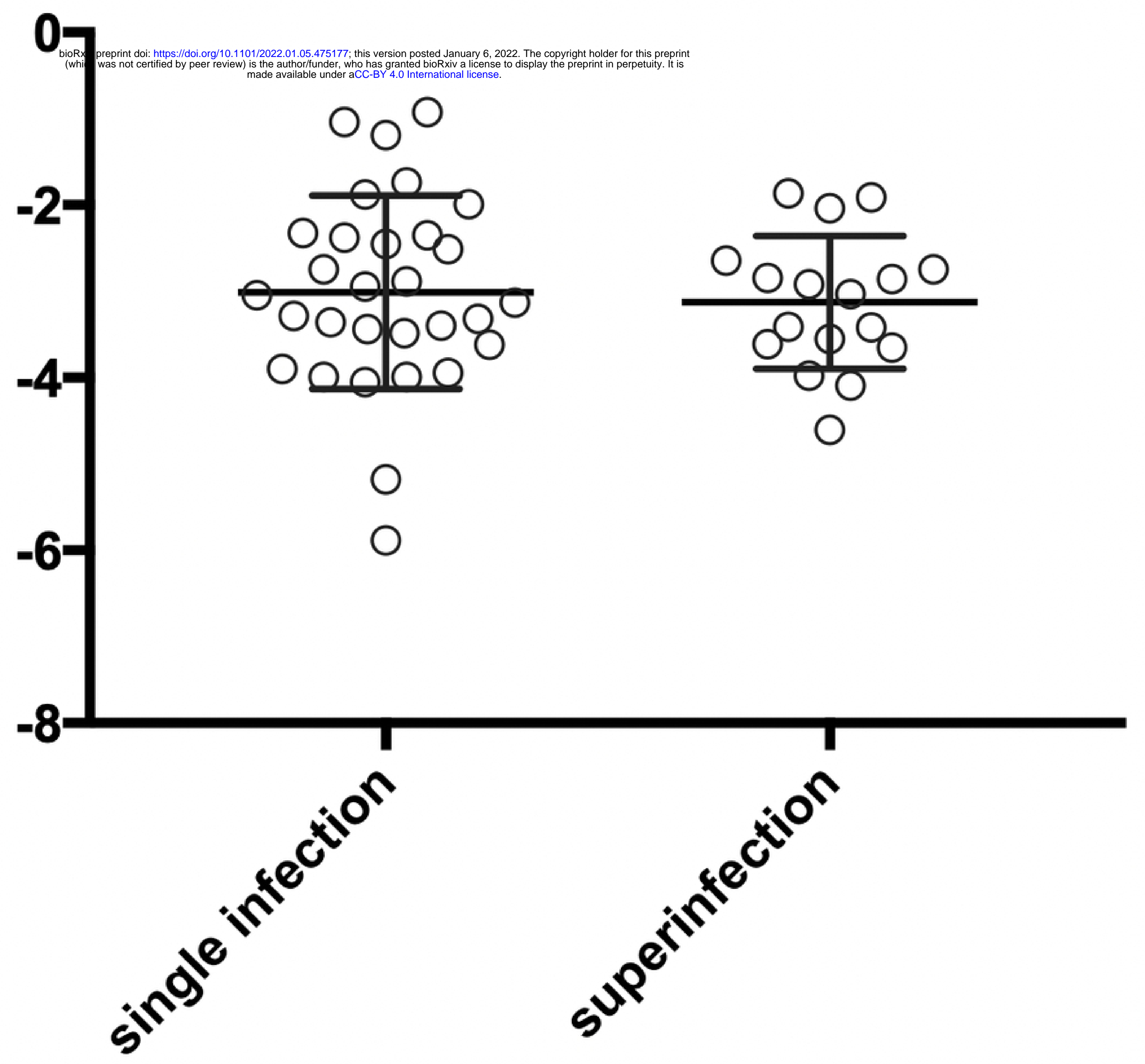

Fig5B 


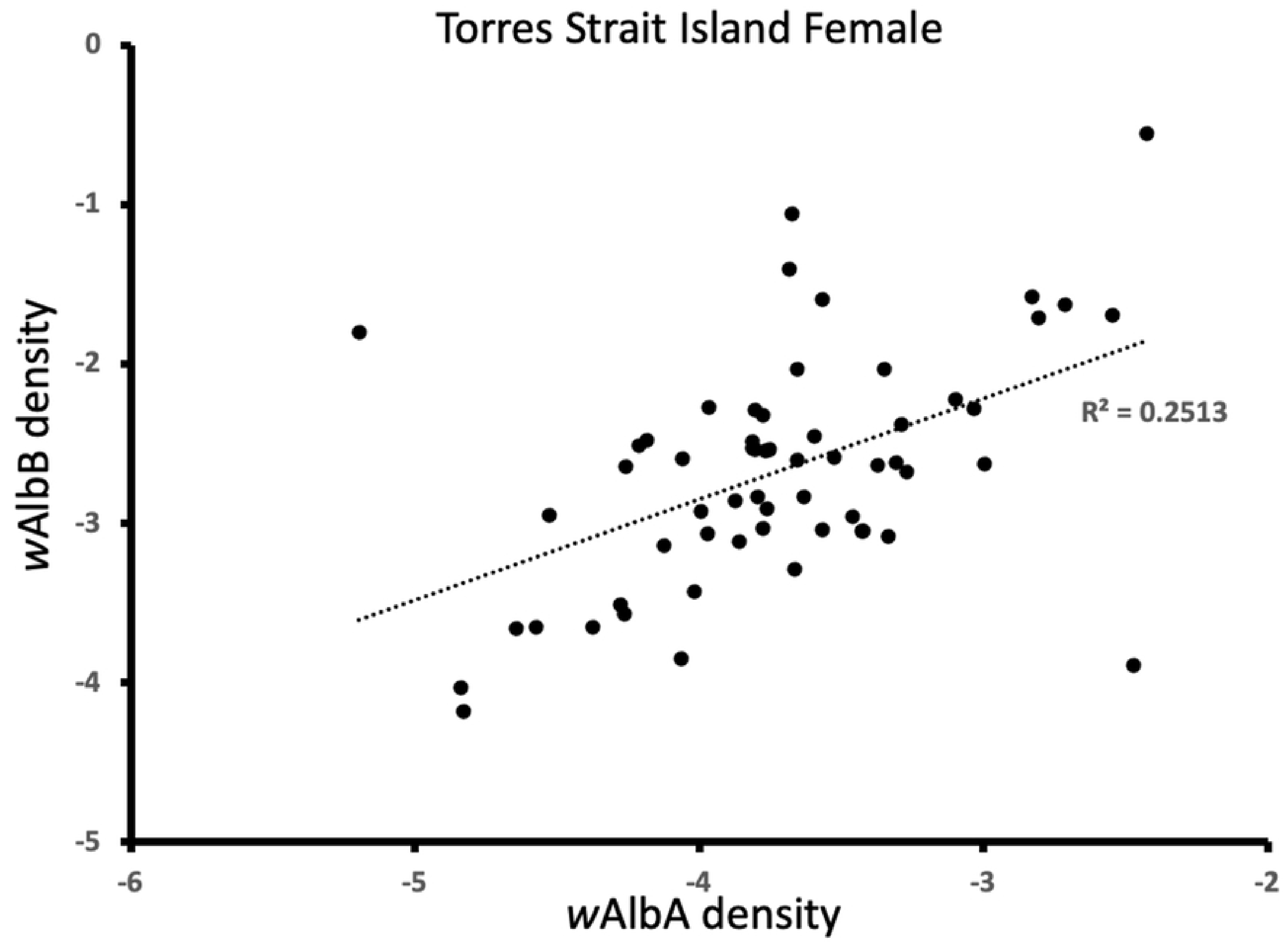

Fig6A 


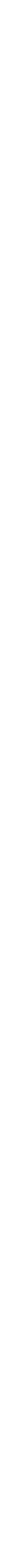

Fig6C 


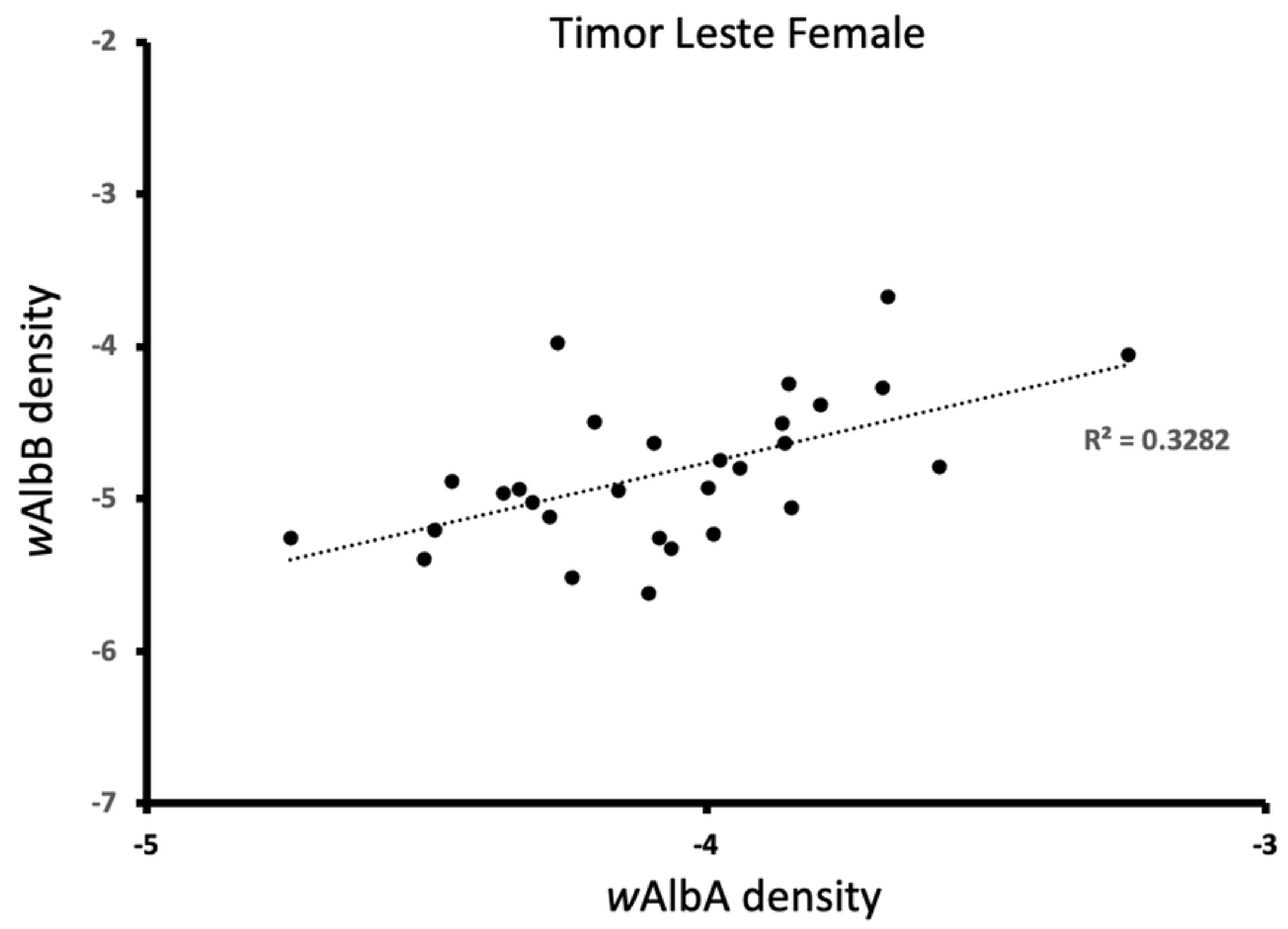

Fig6D 


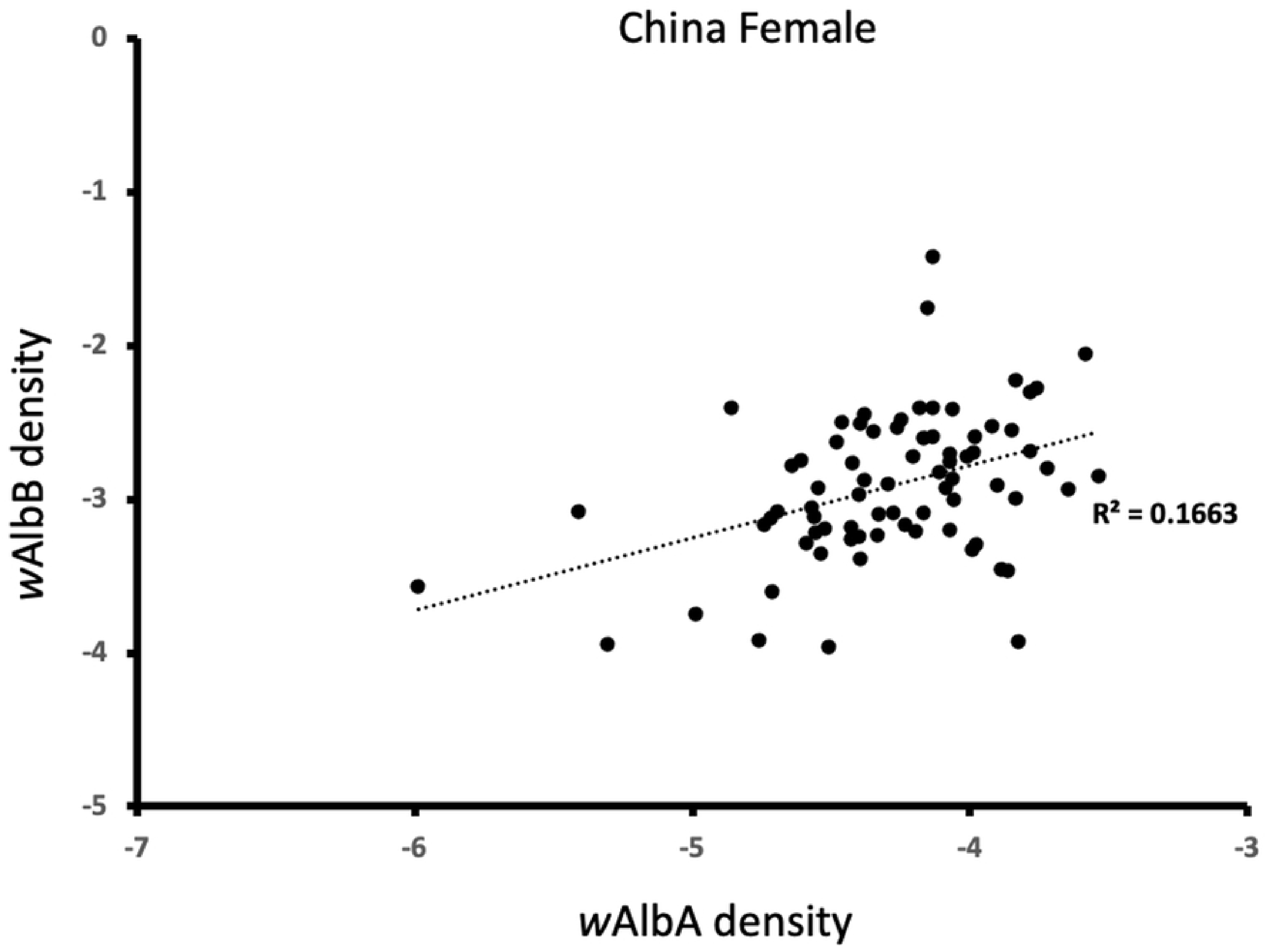

Fig6E 


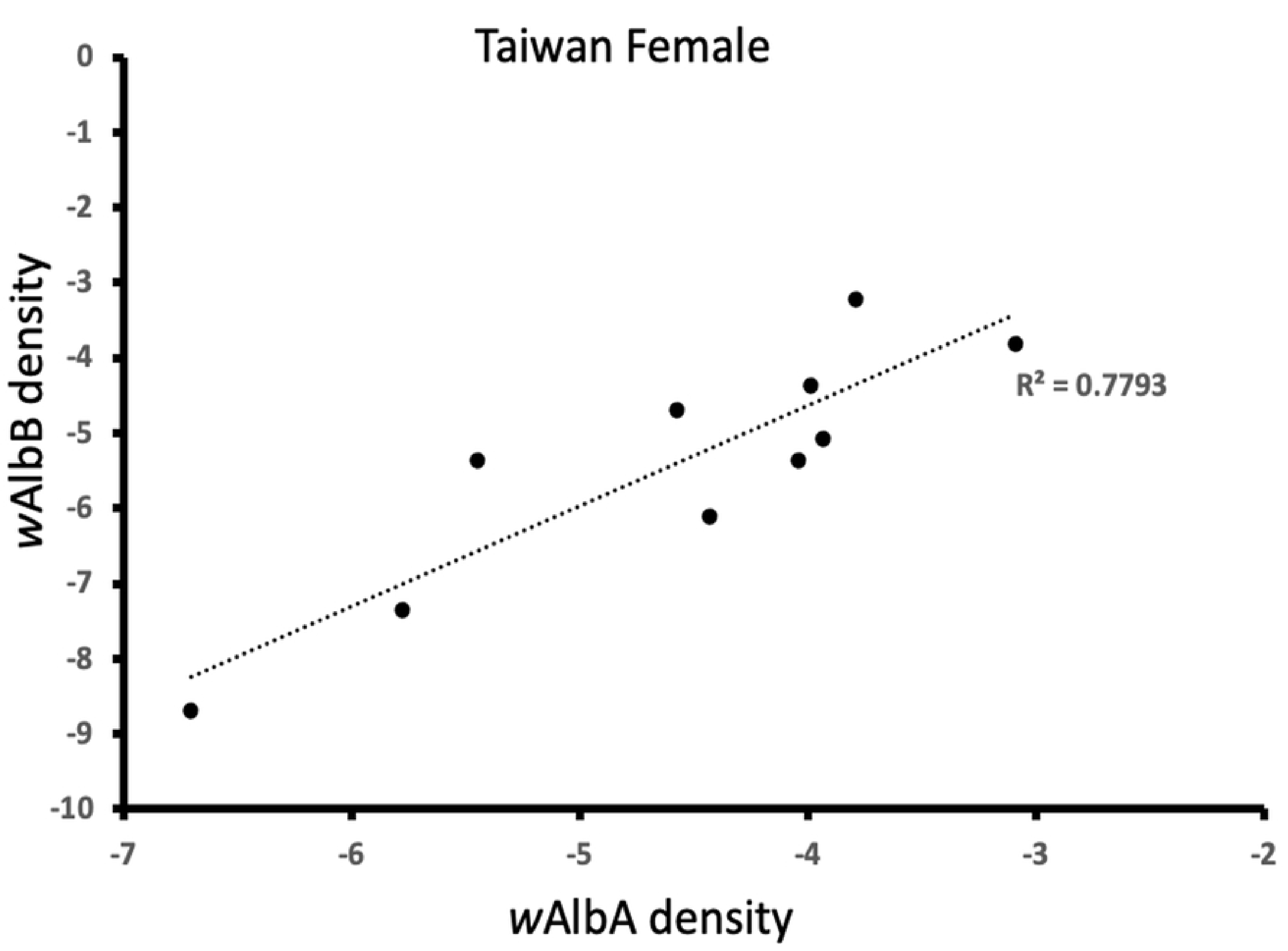

Fig6F 


\section{-1 Torres Strait Island Male}

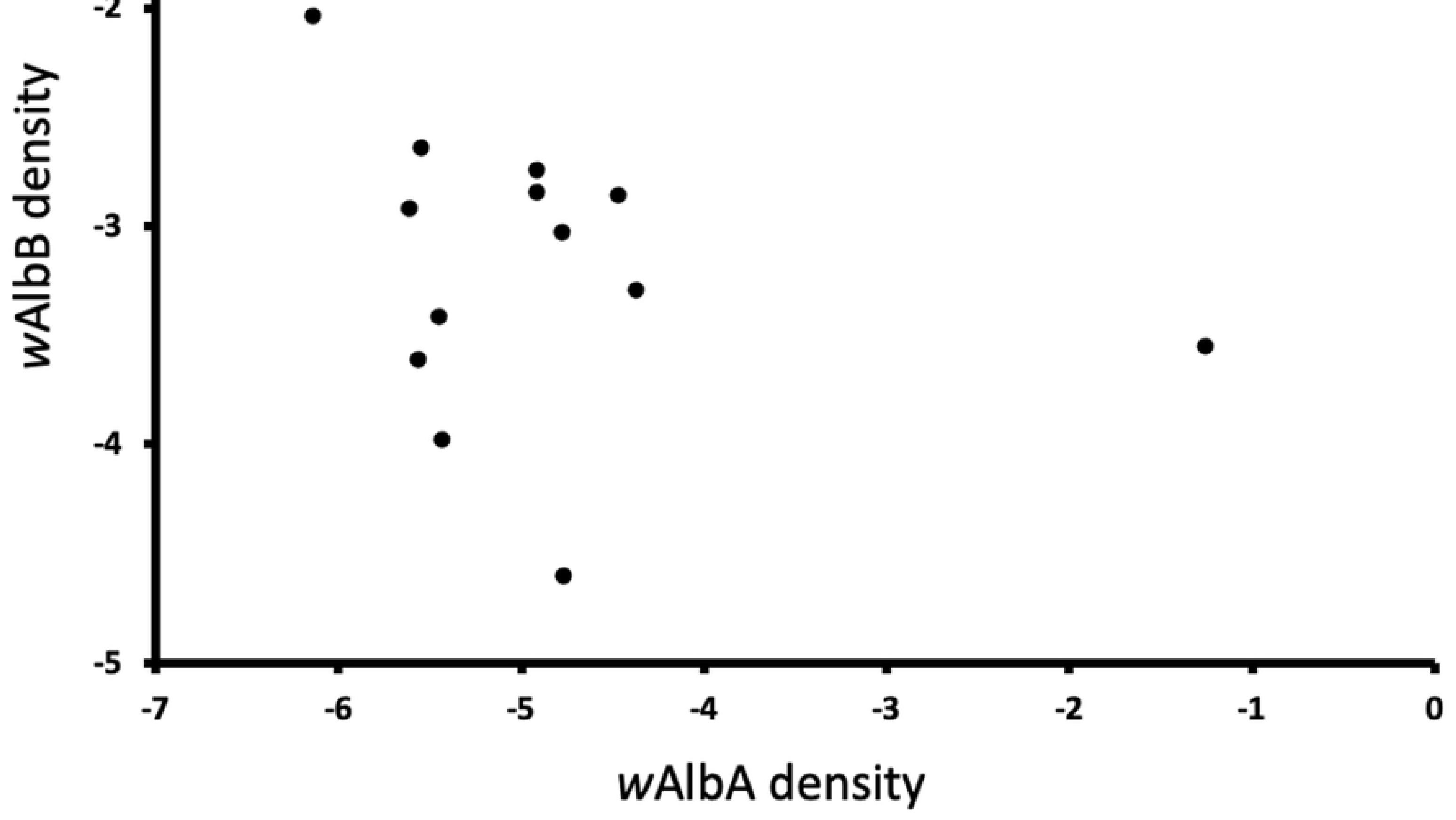

Fig6G 


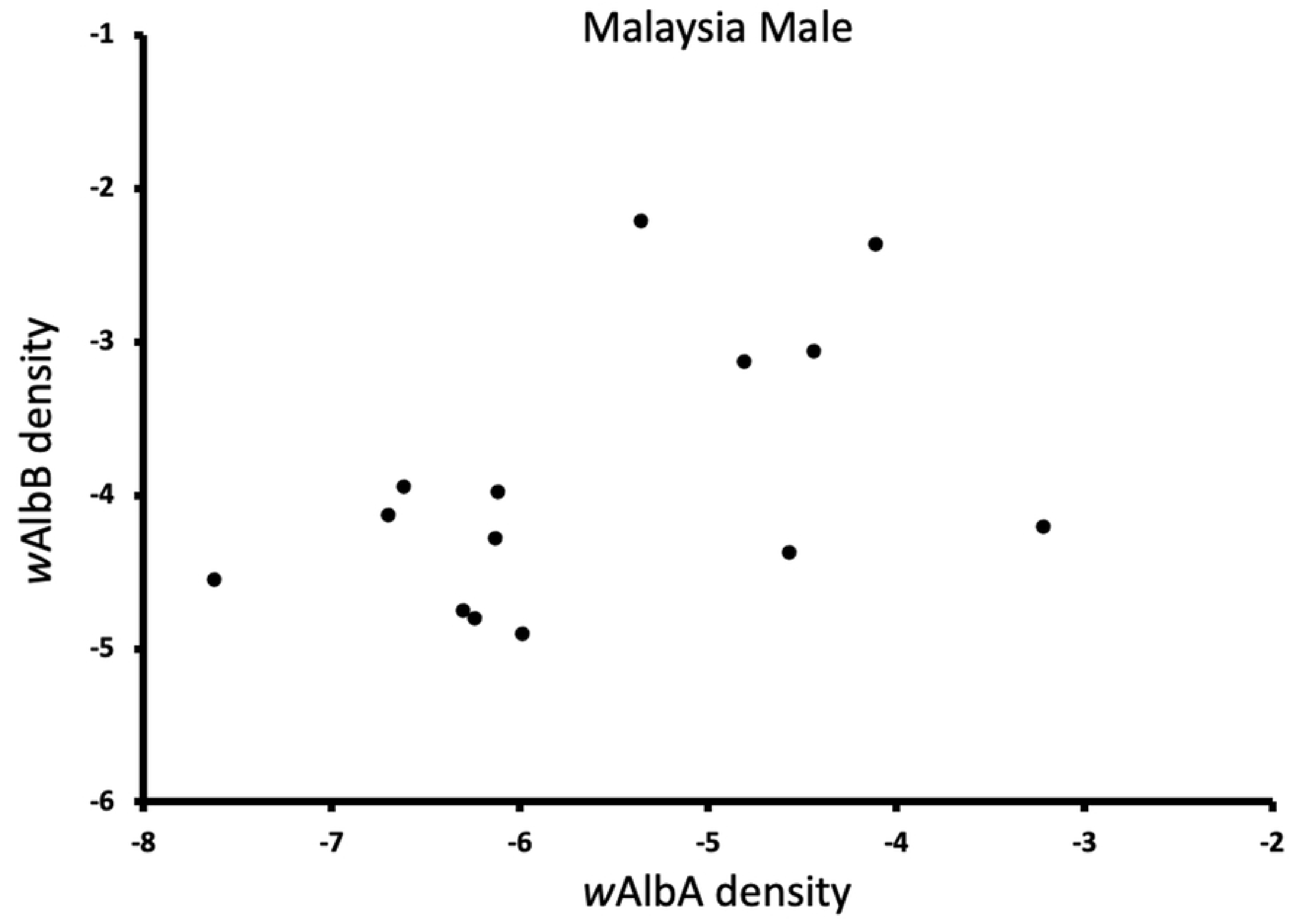

Fig6H 